Pacific

Journal of

Mathematics

LAGRANGE MAPPINGS OF THE FIRST OPEN WHITNEY UMBRELLA

\author{
I.A. Bogaevski and G. IshiKawa
}

Volume $203 \quad$ No. 1

March 2002 


\title{
LAGRANGE MAPPINGS OF THE FIRST OPEN WHITNEY UMBRELLA
}

\author{
I.A. Bogaevski and G. Ishikawa
}

\begin{abstract}
In this paper we give a classification of simple stable singularities of Lagrange projections of the first open Whitney umbrella, the simplest singularity of Lagrange varieties. Our classification extends the $A D E$-classification, due to Arnold, of simple stable singularities of Lagrange projections of smooth Lagrange submanifolds. We also prove a criterion of equivalence of stable Lagrange projections of open Whitney umbrellas which is analogous to Mather's fundamental theorem on stable map-germs.
\end{abstract}

\section{Introduction.}

The systematic investigation of singularities of Lagrange mappings started in 1972 with V.I. Arnold's paper [1]. A Lagrange mapping is the projection of a Lagrange variety in a cotangent fibration onto its base. In the paper [1] it was discovered that singularities of Lagrange mappings of nonsingular Lagrange varieties are classified by degenerations of critical points of smooth functions and the discrete part of their classification is indexed by Coxeter's groups $A_{\mu}, D_{\mu}, E_{\mu}$. This is the so-called $A D E$-classification of simple stable singularities of Lagrange mappings.

However, in applications singular Lagrange varieties appear. Among them open swallow tails and open Whitney umbrellas are very frequently encountered. Open swallow tails occur in the so-called obstacle problem about singularities of the distance on Riemannian manifold with boundary [2], [9], [20], [16]. Concerning open Whitney umbrellas see [8], [9], [13], [15]. They appear naturally in various situations; for instance, as singularities of the generalized Cauchy problems [9], [11], singularities of Riemannian invariants [18], and singularities of tangent developables [19], [20], [14] as the Legendre counterpart. In [9] the discrete part of local classification of Lagrange mappings of open swallow tails is carried out and some singularities of Lagrange mappings of open Whitney umbrellas are found. See also [22].

In this paper, we give the full discrete part of local classification of Lagrange mappings of the open Whitney umbrella of type one, or the first open Whitney umbrella. More accurately, we classify simple stable singularities of mappings of the first open Whitney umbrella. This problem was inspired 
by the classification problem of the composition of an isotropic mapping and a cotangent fibration. (A smooth mapping is called isotropic if the pullback of the symplectic structure is equal to zero.) If the isotropic mapping is an immersion then the discrete part of local classification of the above compositions is the $A D E$-classification. Otherwise, the isotropic mapping can have singularities, first of all, open Whitney umbrellas [8]. Therefore, our classification gives the answer for simple stable compositions of the first open Whitney umbrella and a cotangent fibration.

We recall, in the $A D E$-classification and its generalizations, that stable mappings of Lagrange manifolds are classified by means of families of functions - generating families $[\mathbf{3}],[\mathbf{2 5}]$. Also in our problem, namely, the classification problem of stable projections of Lagrange varieties under Lagrange fibrations, the usage of generating families remains a powerful method, however in a different manner. Namely, we fix a Lagrange variety, while Lagrange fibrations are taken arbitrarily. A Lagrange fibration are regarded as a family of Lagrange submanifolds, and each Lagrange submanifold has a generating function. Thus we describe the Lagrange fibration by means of the family of the generating functions of the Lagrangian fibers, that is, the generating family of the Lagrange fibration. Then we describe stability and simplicity of Lagrange fibrations with respect to symplectic diffeomorphisms preserving the Lagrange variety, by means of the generating families. Similar methods are applied to other problems [23], [24].

First we recall the notions needed to state our main result.

Definition. A smooth fibration $\pi: E \rightarrow Y$ is called Lagrange if its space $E$ is a symplectic manifold and the fibers of $\pi$ are Lagrange submanifolds of E.

For example, the cotangent fibration of a smooth manifold is Lagrange.

We consider the classification problem of singularities on a Lagrange variety under various Lagrange projections. A subset of a symplectic manifold of dimension $2 n$ is called a Lagrange variety if it has a stratification with maximal dimension $n$ such that the symplectic form vanishes on each stratum.

Definition. Let $\Lambda \subset E$ be a Lagrange variety. Two Lagrange fibrations $\pi, \pi^{\prime}: E \rightarrow Y$ are called $\Lambda$-equivalent and denoted by $\pi \sim_{\Lambda} \pi^{\prime}$ if $\pi^{\prime} \circ \tau=\sigma \circ \pi$ where $\sigma$ is a diffeomorphism of $Y$ and $\tau$ is a symplectic diffeomorphism of $E$ which preserves $\Lambda$.

There are two basic notions for the classification problem - stability and simplicity.

Definition. A germ of Lagrange fibration $\pi:\left(E, z_{0}\right) \rightarrow Y$ is called stable with respect to $\Lambda$-equivalence, or $\Lambda$-stable, if for any sufficiently small Lagrange perturbation of any its representative $\widetilde{\pi}: U \rightarrow Y, z_{0} \in U \subset E$ 
there exists a point $z \in U$ such that the germ of the perturbation at $z$ is $\Lambda$-equivalent to the original germ $\pi$. (See $[\mathbf{9}],[\mathbf{1 5}]$.)

Definition. A germ of Lagrange fibration $\pi:\left(E, z_{0}\right) \rightarrow Y$ is called simple with respect to $\Lambda$-equivalence, or $\Lambda$-simple, if there exists its representative $\tilde{\pi}: U \rightarrow Y, z_{0} \in U \subset E$ such that the number of $\Lambda$-equivalence classes of the germs $(U, z) \rightarrow Y$ for all $z \in U$ of all sufficiently small Lagrange perturbations of $\tilde{\pi}$ is finite.

Let $\Lambda_{1}$ be the first open Whitney umbrella given by the following parametric form:

$$
p_{1}=x_{2} t, \quad p_{2}=t^{3} / 3, \quad p_{3}=\cdots=p_{n}=0, \quad x_{1}=t^{2} / 2,
$$

where $t$ is the parameter, $(p, x)=\left(p_{1}, \ldots, p_{n}, x_{1}, \ldots, x_{n}\right)$ are local coordinates in $E$ such that $\omega=d p \wedge d x$, and $n \geq 2$. See [8], [9], [13], [15].

Then $\Lambda_{1}$ is an $n$-dimensional algebraic Lagrange variety (Lemma 3 ), whose singular locus $\Sigma\left(\Lambda_{1}\right)=\left\{p=0, x_{1}=0, x_{2}=0\right\}$ is a nonsingular submanifold of $E$ of dimension $n-2$.

Remark that, at a regular point $z_{0} \in \Lambda_{1}$, there exists a system of symplectic coordinates $(p, x)$ around $\left(E, z_{0}\right)$ such that $\Lambda_{1}$ is defined by $\left\{p_{1}=\right.$ $\left.0, \ldots, p_{n}=0\right\}$.

Now a Lagrange fibration is given by a family of Lagrange submanifolds. It is well-known that a smooth Lagrange submanifold is locally given by $x_{I}=w_{p_{I}}\left(p_{I}, x_{J}\right), p_{J}=-w_{x_{J}}\left(p_{I}, x_{J}\right)$, where $I, J$ is a decomposition of $\{1,2, \ldots, n\}$ and $w\left(p_{I}, x_{J}\right)$ is a smooth function.

Definition. Let $W: \mathbb{R}^{n} \times \mathbb{R}^{n} \rightarrow \mathbb{R}$ be a smooth function of $p_{I}, x_{J}$, and $y=\left(y_{1}, \ldots, y_{n}\right)$, which satisfies the condition of nondegeneracy:

$$
\operatorname{det}\left\|\begin{array}{c}
W_{p_{I} y} \\
W_{x_{J} y}
\end{array}\right\| \neq 0
$$

where $I \cap J=\emptyset, I \cup J=\{1, \ldots, n\}$. Then $W$ is called a generating family of the Lagrange fibration $\pi:(p, x) \mapsto y$ whose symplectic structure and fibers are given by the formulas

$$
\omega=d p \wedge d x, \quad \pi^{-1}(y)=\left\{x_{I}=W_{p_{I}}\left(p_{I}, x_{J}, y\right), p_{J}=-W_{x_{J}}\left(p_{I}, x_{J}, y\right)\right\} .
$$

(Locally $\pi$ is a fibration in consequence of nondegeneracy of $W$.)

For example, the natural Lagrange projections $(p, x) \mapsto p$ and $(p, x) \mapsto x$ are given by the generating families $-x y$ and $p y$ respectively.

Then the main result of this paper is the following:

Theorem 1. Let us consider a germ of a Lagrange fibration at a point of the first open Whitney umbrella $\Lambda_{1}$. If our germ is simple and stable with respect to $\Lambda_{1}$-equivalence then it is $\Lambda_{1}$-equivalent to the germ at the origin of the fibration defined by one of the following generating families: 
Classification of simple stable projections at regular points $\Lambda_{1}=$ $\left\{p_{1}=\cdots=p_{n}=0\right\}$.

$\left.A_{1}\right) W\left(p_{1}, \ldots, p_{n}, y\right)=y_{1} p_{1}+\cdots+y_{n} p_{n}$;

$\left.A_{m}^{ \pm}\right) W\left(x_{1}, p_{2}, \ldots, p_{n}, y\right)= \pm x_{1}^{m+1}+y_{2} x_{1}^{m-1}+\cdots+y_{m-1} x_{1}^{2}-y_{1} x_{1}+y_{2} p_{2}+$ $\cdots+y_{n} p_{n}$, where $n \geq m-1 \geq 1$ and $A_{m}^{+} \sim_{\Lambda_{1}} A_{m}^{-}$if $m$ is even;

$\left.D_{m}^{ \pm}\right) W\left(x_{1}, x_{2}, p_{3}, \ldots, p_{n}, y\right)=x_{1}^{2} x_{2} \pm x_{2}^{m-1}+y_{3} x_{2}^{m-2}+\cdots+y_{m-1} x_{2}^{2}-y_{1} x_{1}-$ $y_{2} x_{2}+y_{3} p_{3}+\cdots+y_{n} p_{n}$, where $n \geq m-1 \geq 3$;

$\left.E_{6}^{ \pm}\right) W\left(x_{1}, x_{2}, p_{3}, \ldots, p_{n}, y\right)=x_{1}^{3} \pm x_{2}^{4}+y_{3} x_{1} x_{2}^{2}+y_{4} x_{1} x_{2}+y_{5} x_{2}^{2}-y_{1} x_{1}-$ $y_{2} x_{2}+y_{3} p_{3}+\cdots+y_{n} p_{n}$, where $n \geq 5$

$\left.E_{7}\right) W\left(x_{1}, x_{2}, p_{3}, \ldots, p_{n}, y\right)=x_{1}^{3}+x_{1} x_{2}^{3}+y_{3} x_{1} x_{2}^{2}+y_{4} x_{1} x_{2}+y_{5} x_{2}^{3}+y_{6} x_{2}^{2}-$ $y_{1} x_{1}-y_{2} x_{2}+y_{3} p_{3}+\cdots+y_{n} p_{n}$, where $n \geq 6$;

$\left.E_{8}\right) W\left(x_{1}, x_{2}, p_{3}, \ldots, p_{n}, y\right)=x_{1}^{3}+x_{2}^{5}+y_{3} x_{1} x_{2}^{3}+y_{4} x_{1} x_{2}^{2}+y_{5} x_{1} x_{2}+y_{6} x_{2}^{3}+$ $y_{7} x_{2}^{2}-y_{1} x_{1}-y_{2} x_{2}+y_{3} p_{3}+\cdots+y_{n} p_{n}$, where $n \geq 7$.

\section{Classification of simple stable projections at singular points.}

$\left.S_{3}\right) W(p, y)=y_{1} p_{1}+\cdots+y_{n} p_{n}$, where $n \geq 2$;

$\left.S_{m}^{ \pm}\right) W\left(x_{1}, p_{2}, \ldots, p_{n}, y\right)= \pm e_{m+1}+y_{3} e_{4}+\cdots+y_{m-1} e_{m}+y_{1} x_{1}+y_{2} p_{2}+$ $\cdots+y_{n} p_{n}$, where $e_{2 k}=x_{1}^{k}, e_{2 k+1}=x_{1}^{k-1} p_{2}, n \geq m-1 \geq 3$, and $S_{m}^{+} \sim_{\Lambda_{1}} S_{m}^{-}$if $m$ is even;

$\left.T_{5}\right) W\left(p_{1}, x_{2}, p_{3}, \ldots, p_{n}, y\right)=x_{2}^{3}+y_{3} p_{1} x_{2}+y_{4} x_{2}^{2}+y_{1} p_{1}+y_{2} x_{2}+y_{3} p_{3}+$ $\cdots+y_{n} p_{n}$, where $n \geq 4$

U6) $W\left(x_{1}, x_{2}, p_{3}, \ldots, p_{n}, y\right)=x_{2}^{3}+y_{3} x_{1}^{2}+y_{4} x_{1} x_{2}+y_{5} x_{2}^{2}+y_{1} x_{1}+y_{2} x_{2}+$ $y_{3} p_{3}+\cdots+y_{n} p_{n}$, where $n \geq 5$

$\left.V_{6}\right) W\left(p_{1}, p_{2}, x_{3}, p_{4}, \ldots, p_{n}, y\right)=x_{3}^{3}+y_{4} p_{1} x_{3}+y_{5} p_{2} x_{3}+y_{1} p_{1}+y_{2} p_{2}+y_{3} x_{3}+$ $y_{4} p_{4}+\cdots+y_{n} p_{n}$, where $n \geq 5$.

Generic Lagrange fibration is stable with respect to $\Lambda_{1}$-equivalence if $n \leq$ 4. If $n>4$, there exists a Lagrange fibration such that any its sufficient small perturbation has an unstable germ.

Remark. The change $\left(t, p_{2}, x_{2}\right) \mapsto-\left(t, p_{2}, x_{2}\right)$ shows us that $S_{m}^{+} \sim_{\Lambda_{1}} S_{m}^{-}$if $m$ is even.

We examine examples from the list of Theorem 1 .

(1) The singularity $S_{4}, n=3$. The generating family is given by

$$
W=x_{1} p_{2}+y_{3} x_{1}^{2}+y_{1} x_{1}+y_{2} p_{2}+y_{3} p_{3},
$$

and the Lagrange fibration is given by

$$
\left\{\begin{array}{l}
y_{1}=-p_{1}-p_{2}-2 x_{1} x_{3} \\
y_{2}=-x_{1}+x_{2} \\
y_{3}=x_{3}
\end{array}\right.
$$


The composition with the parametrization of the open Whitney umbrella is given by

$$
\left\{\begin{array}{l}
y_{1}=-x_{2} t-\frac{t^{3}}{3}-x_{3} t^{2} \\
y_{2}=-\frac{t^{2}}{2}+x_{2} \\
y_{3}=x_{3} .
\end{array}\right.
$$

The caustic of the singularity $S_{4}$ is a surface with a cuspidal edge (Figure 1). The edge consists of $A_{3}$-points and the $S_{4}$-point (the origin). The tangent line to the edge at the origin consists of $S_{3}$-points and the origin. In fact, the caustic is the tangent developable of the cuspidal edge, consisting of the tangent lines to the cuspidal edge.

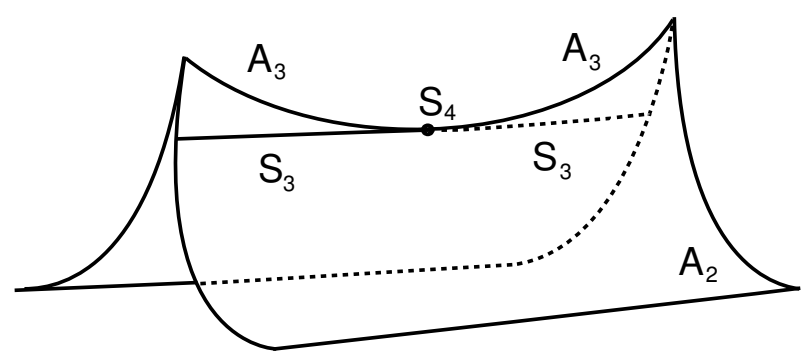

Figure 1. $S_{4}$-caustic.

(2) The singularity $T_{5}, n=4$. The generating family is given by

$$
W\left(p_{1}, x_{2}, p_{3}, p_{4}, y\right)=x_{2}^{3}+y_{3} p_{1} x_{2}+y_{4} x_{2}^{2}+y_{1} p_{1}+y_{2} x_{2}+y_{3} p_{3}+y_{4} p_{4} \text {. }
$$

Then the Lagrange fibration is given by

$$
\left\{\begin{array}{l}
y_{1}=x_{1}-x_{2} x_{3} \\
y_{2}=-p_{2}-3 x_{2}^{2}-x_{3} p_{1}-2 x_{2} x_{4} \\
y_{3}=x_{3} \\
y_{4}=x_{4}
\end{array}\right.
$$

and the composition with the parametrization of $\Lambda_{1}$ is given by

$$
\left\{\begin{array}{l}
y_{1}=\frac{t^{2}}{2}-x_{2} x_{3} \\
y_{2}=-\frac{t^{3}}{3}-3 x_{2}^{2}-x_{2} x_{3} t-2 x_{2} x_{4} \\
y_{3}=x_{3} \\
y_{4}=x_{4}
\end{array}\right.
$$

This provides the example of stable projection of corank two at the singular point in the smallest dimension. 
Recall that, in the general singularity theory of mappings, the classification of $C^{\infty}$-stable map-germs is reduced to their classification up to the contact equivalence [17] IV. Also recall that, in Lagrange singularity theory, the Lagrange classification of Lagrange stable immersion-germs is reduced to the classification of function-germs up to the right equivalence. Naturally, in our classification problem, we need an analogous result to establish the actual classification.

Definition. Let $\pi:\left(E, z_{0}\right) \rightarrow\left(Y, y_{0}\right)$ be a germ of Lagrange fibration. We call the germ of Lagrange submanifold $\left(\pi^{-1}\left(y_{0}\right), z_{0}\right) \subset E$ the central fiber of the germ $\pi$.

Definition. Let $\Lambda \subset E$ be a Lagrange variety. Two germs of Lagrange submanifolds $L, L^{\prime} \subset E$ are called $\Lambda$-equivalent and denoted by $L \sim_{\Lambda} L^{\prime}$ if they are the same with respect to a symplectic diffeomorphism preserving $\Lambda$, namely if there exists a symplectic diffeomorphism $\tau: E \rightarrow E$ such that $\tau(\Lambda)=\Lambda$ and that $\tau(L)=L^{\prime}$.

Definition. The germs $L$ and $L^{\prime}$ are called formally $\Lambda$-equivalent and denoted by $j^{\infty}(L) \sim_{\Lambda} j^{\infty}\left(L^{\prime}\right)$ if their $\infty$-jets are the same with respect to a symplectic diffeomorphism preserving $\Lambda$. More accurately, $j^{\infty}(L) \sim_{\Lambda}$ $j^{\infty}\left(L^{\prime}\right)$ if there exist parametrizations $i, i^{\prime}:\left(\mathbb{R}^{n}, 0\right) \rightarrow E$ of $L, L^{\prime}$ respectively, and a symplectic diffeomorphism $\tau: E \rightarrow E$ preserving $\Lambda$ such that $j^{\infty} i^{\prime}(0)=j^{\infty}(\tau \circ i)(0)$.

We say that $\Lambda \subset E$ is an open Whitney umbrella if $\Lambda$ is the image of an open Whitney umbrella $f_{n, k}:\left(\mathbb{R}^{n}, 0\right) \rightarrow(E, 0)$ in the sense of $[\mathbf{1 3}]$. We have open Whitney umbrellas $\Lambda_{k}, 0 \leq k \leq[n / 2] ; \Lambda_{0}$ is a Lagrange submanifold and $\Lambda_{1}$ is the first open Whitney umbrella already introduced.

Then we prove and use in this paper the following:

Theorem 2. Let $\Lambda \subset E$ be an open Whitney umbrella. Then two $\Lambda$-stable germs of Lagrange fibrations $\pi, \pi^{\prime}: E \rightarrow Y$ are $\Lambda$-equivalent if and only if their central fibers are $\Lambda$-equivalent. Moreover, $\pi$ and $\pi^{\prime}$ are $\Lambda$-equivalent if and only if their central fibers are formally $\Lambda$-equivalent.

Consider the case when $\Lambda$ is a Lagrange submanifold $(k=0)$. Then we may take symplectic coordinates $(p, x)$ with $\Lambda=\{x=0\}$. Recall the fundamental theorem of Lagrange singularity theory $[\mathbf{3}]$ : Two germs of Lagrange submanifolds in $T^{*} \mathbb{R}^{n}$ are Lagrange equivalent for the canonical projection $T^{*} \mathbb{R}^{n} \rightarrow \mathbb{R}^{n}$ if and only if their generating families are stably $R^{+}$-equivalent. Moreover, a Lagrange submanifold is Lagrange stable if and only if its generating family $F(q, x), q$ being the inner variables, is an $R^{+}$-versal deformation of $F(q, 0)[3]$. So, two Lagrange stable Lagrange submanifold are Lagrange equivalent if their generating families are deformations of stably $R^{+}$-equivalent function germs. Besides, we recall the notion of "contact 
equivalence" for Lagrange manifolds due to Golubitsky and Guillemin [10]. Then two germs $L, L^{\prime}$ of Lagrange submanifolds are contact equivalent via a symplectic diffeomorphism in the sense [10] if and only if $L, L^{\prime}$ are $\Lambda$ equivalent in our sense. Let $L, L^{\prime}$ be the graphs of the differentials of function germs $h(x), h^{\prime}(x)$ which have an order $\geq 3$. Then the germs $L$ and $L^{\prime}$ are $\Lambda$-equivalent if and only if $h$ and $h^{\prime}$ are right equivalent ([10], Prop. 4.2.). In general $L, L^{\prime}$ are $\Lambda$-equivalent if and only if $F(q, 0)$ and $F^{\prime}\left(q^{\prime}, 0\right)$ are stably $R^{+}$-equivalent, for the generating families $F(q, x), F\left(q^{\prime}, x\right)$ of $L, L^{\prime}$ respectively. Therefore, Theorem 2 is a quite natural generalization of the fundamental theorem of Lagrange singularity theory. Also it is a Lagrange counterpart of the Mather's theorem "two $\mathcal{A}$-stable mappings are $\mathcal{A}$-equivalent if and only if they are $\mathcal{K}$-equivalent" $[\mathbf{1 7}]$ in ordinary singularity theory of stable mappings. See also [15] $\S 6$.

In the next section we give the formal classification of central fibers of simple stable projections of the first open Whitney umbrella $\Lambda_{1}$ up to $\Lambda_{1^{-}}$ preserving symplectic diffeomorphisms (Theorem 3). Theorem 3 follows from Theorem 4 which is reduced to technically key Lemma 1 . In $\S 2$, we prepare Lemmas needed in the following section. In particular, we give explicit equations defining the first open Whitney umbrella. Then Lemma 1 is proved in $\S 3$. Theorems 2 and 1 are proved in $\S 4$. In $\S 5$ we describe relations between our study and simple stable compositions.

For the proof of Theorem 1 we use explicit equations defining the first open Whitney umbrella. In order to carry out classifications of simple stable projections of general open Whitney umbrellas applying the method used in the present paper, we need to get explicit equations for them. That problem is left open.

The authors would like to thank the referee for the helpful comments.

\section{Normal forms of fibers.}

We start to prove Theorem 1 with finding formal normal forms for separate fibers which pass through singular points of the first open Whitney umbrella $\Lambda_{1}$ with respect to symplectic diffeomorphisms preserving $\Lambda_{1}$ itself.

Definition. A germ of Lagrange submanifold $L \subset E$ is called simple with respect to $\Lambda$-equivalence, or $\Lambda$-simple, if the number of $\Lambda$-equivalence classes of all germs of the kind $\tau(L)$ is finite, where $\tau: E \rightarrow E$ is any sufficiently small symplectic perturbation of the identity diffeomorphism.

In particular, a $\Lambda$-stable germ of Lagrange fibration is called simple with respect to $\Lambda$-equivalence, or $\Lambda$-simple, if there exists its representative such that the number of $\Lambda$-equivalence classes of all its germs is finite.

In coordinates $(p, x)$ such that $\omega=d p \wedge d x$, any Lagrange submanifold is locally given by at least one of the $2^{n}$ generating functions $w\left(p_{I}, x_{J}\right)$ by the 
formulas:

$$
x_{I}=w_{p_{I}}\left(p_{I}, x_{J}\right), \quad p_{J}=-w_{x_{J}}\left(p_{I}, x_{J}\right)
$$

where $I \cap J=\emptyset$ and $I \cup J=\{1, \ldots, n\}$.

Theorem 3. Let us consider a germ of a Lagrange submanifold at a singular point of the first open Whitney umbrella $\Lambda_{1}$. If our germ is $\Lambda_{1}$-simple then it is formally $\Lambda_{1}$-equivalent to the germ at the origin of the Lagrange submanifold defined by one of the following generating functions:

$\left.S_{3}\right) w\left(p_{1}, \ldots, p_{n}\right)=0$;

$\left.S_{m}^{ \pm}\right) w\left(x_{1}, p_{2}, \ldots, p_{n}\right)= \pm e_{m+1}$ where $e_{2 k}=x_{1}^{k}, e_{2 k+1}=x_{1}^{k-1} p_{2}, m \geq 4$, $S_{m}^{+} \sim_{\Lambda_{1}} S_{m}^{-}$if $m$ is even;

$\left.T_{5}\right) w\left(p_{1}, x_{2}, p_{3}, \ldots, p_{n}\right)=x_{2}^{3}$;

$\left.U_{6}\right) w\left(x_{1}, x_{2}, p_{3}, \ldots, p_{n}\right)=x_{2}^{3}$;

$\left.V_{6}\right) w\left(p_{1}, p_{2}, x_{3}, p_{4}, \ldots, p_{n}\right)=x_{3}^{3}$ where $n \geq 3$.

Non-simple germs occur in families of Lagrange submanifolds depending generically on at least 5 parameters. In generic 4-parametric families such germ do not occur.

Remark. The change $\left(t, p_{2}, x_{2}\right) \mapsto-\left(t, p_{2}, x_{2}\right)$ shows us that $S_{m}^{+} \sim_{\Lambda_{1}} S_{m}^{-}$if $m$ is even.

Proof. Theorem 3 follows from the following Theorem 4.

Definition. Let $\Lambda \subset E$ be a Lagrange variety. Two germs $w, w^{\prime}$ of generating functions are called formally $\Lambda$-equivalent and denoted by $w \sim_{\Lambda} w^{\prime}$ if the corresponding germs of Lagrange submanifolds are formally $\Lambda$-equivalent.

\section{Theorem 4.}

I. A germ of a Lagrange submanifold at a singular point of the first open Whitney umbrella $\Lambda_{1}$ is $\Lambda_{1}$-equivalent to the germ at the origin of the Lagrange submanifold defined by a generating function $w\left(p_{I}, x_{J}\right)$ such that $w_{p_{I}}(0)=w_{x_{J}}(0)=w_{x_{J} x_{J}}(0)=0$ and one of the following conditions is satisfied:

1) $J=\emptyset(c=2)$;

2) $J=\{1\}(c=3)$;

3) $J=\{2\}, w_{p_{1} x_{2}}(0)=0, w_{x_{2} x_{2} x_{2}}(0) \neq 0(c=4)$;

4) $J=\{2\}, w_{p_{1} x_{2}}(0)=w_{x_{2} x_{2} x_{2}}(0)=0(c=5)$;

5) $J=\{3\}, w_{p_{1} x_{3}}(0)=w_{p_{2} x_{3}}(0)=0, w_{x_{3} x_{3} x_{3}}(0) \neq 0(c=5)$;

6) $J=\{3\}, w_{p_{1} x_{3}}(0)=w_{p_{2} x_{3}}(0)=w_{x_{3} x_{3} x_{3}}(0)=0(c=6)$;

7) $J=\{1,2\}, w_{x_{2} x_{2} x_{2}}(0) \neq 0(c=5)$;

8) $J=\{1,2\}, w_{x_{2} x_{2} x_{2}}(0)=0(c=6)$;

9) $J=\{1,3\}, w_{p_{2} x_{3}}(0)=0(c=6)$;

10) $J=\{2,3\}, w_{p_{1} x_{2}}(0)=w_{p_{1} x_{3}}(0)=0(c=7)$;

11) $J=\{3,4\}, w_{p_{1} x_{3}}(0)=w_{p_{1} x_{4}}(0)=w_{p_{2} x_{3}}(0)=w_{p_{2} x_{4}}(0)=0(c=9)$;

12) $\# J \geq 3(c=8)$. 
Such germs occur in families of Lagrange submanifolds depending generically on at least c parameters. Case 8 is adjacent to Case 4; Cases 10 and 11 are adjacent to Case 9.

II. In the above cases:

1) $w \sim \Lambda_{1} 0$;

2) $w$ is not $\Lambda_{1}$-simple or $\exists m \geq 4$ such that $w \sim_{\Lambda_{1}} \pm e_{m+1}$ where $e_{2 k}=x_{1}^{k}, e_{2 k+1}=x_{1}^{k-1} p_{2}$

3) $w \sim \Lambda_{1} x_{2}^{3}$;

7) $w \sim \Lambda_{1} x_{2}^{3}$;

5) $w \sim \Lambda_{1} x_{3}^{3}$;

4,6,8-12) $w$ is not $\Lambda_{1}$-simple.

The non- $\Lambda_{1}$-simple germs from Case 2 have infinite codimension. They are adjacent to $S_{m}^{ \pm}$.

Proof. I. The singularities of the first open Whitney umbrella $\Lambda_{1}$ are defined by the equations $p_{1}=\cdots=p_{n}=x_{1}=x_{2}=0$. After a shift we get $w_{p_{I}}(0)=w_{x_{J}}(0)=0$. If some principal minor det $\left\|w_{x_{J^{\prime}} x_{J^{\prime}}}(0)\right\| \neq 0$ where $J^{\prime} \subset J$, then we can change $I \mapsto I \cup J^{\prime}$ and $J \mapsto J \backslash J^{\prime}$. Therefore, we assume $w_{x_{J} x_{J}}(0)=0$. After renumbering among $p_{3}, \ldots, p_{n}$ we reach one (but not only one) of the following cases: $J=\emptyset, J=\{1\}, J=\{2\}, J=\{3\}$, $J=\{1,2\}, J=\{1,3\}, J=\{2,3\}, J=\{3,4\}$, or $\# J \geq 3$. In order to eliminate some of these cases let us note that we can replace $i \mapsto j$ in $I$ and $j \mapsto i$ in $J$ if $w_{p_{i} x_{j}}(0) \neq 0$ where $i \in I$ and $j \in J$.

The singularities of the first open Whitney umbrella $\Lambda_{1}$ form a submanifold of codimension $n+2$. So, germs passing through singularities of $\Lambda_{1}$ occur in families of Lagrange submanifolds depending generically on at least 2 parameters. This is the case $J=0$. The other cases require the extra number of parameters which is equal to the quantity of conditions for the second and third derivatives.

II. It is sufficient to prove Cases 1, 2, 3, 7 for $n=2$ and Case 5 for $n=3$. This follows from the equivalence $w \sim_{\Lambda_{1}} w_{0}$ where $w_{0}\left(p_{I}, x_{J}\right)=\left.w\right|_{p_{I^{\prime \prime}}=0}$, $I^{\prime}=I \cap\{1,2\}$, and $I^{\prime \prime}=I \cap\{3, \ldots, n\}$. The equivalence is performed by the symplectic diffeomorphism

$$
\left(p_{I}, p_{J}, x_{I}, x_{J}\right) \mapsto\left(p_{I}, p_{J}+\widetilde{w}_{x_{J}}, x_{I}-\widetilde{w}_{p_{I}}, x_{J}\right)
$$

where $\widetilde{w}=w-w_{0}$. This diffeomorphism preserves $\Lambda_{1}$ because it shifts the plane $p_{I^{\prime \prime}}=0$ along only $x_{I^{\prime \prime}}$ (preserving $p_{I}, p_{J}, x_{I^{\prime}}$, and $x_{J}$ ) that follows from the equality $\left.\widetilde{w}\right|_{p_{I^{\prime \prime}}=0}=0$.

The following infinite chains

$$
\begin{aligned}
& 1_{2} \Rightarrow 1_{3} \Rightarrow \ldots \\
2_{5} \Rightarrow 2_{6} \Rightarrow \ldots \quad \text { or } \quad 2_{5} \Rightarrow 2_{6} & \Rightarrow \cdots \Rightarrow 2_{m+1} \Rightarrow 2_{m+1}^{m+2} \Rightarrow 2_{m+1}^{m+3} \Rightarrow \ldots, \\
3_{6} & \Rightarrow 3_{6}^{7} \Rightarrow 3_{6}^{8} \Rightarrow \ldots
\end{aligned}
$$




$$
\begin{gathered}
5_{6} \Rightarrow 5_{6}^{7} \Rightarrow 5_{6}^{8} \Rightarrow \ldots, \\
7_{3} \Rightarrow 7_{3}^{4} \Rightarrow 7_{3}^{5} \Rightarrow \ldots
\end{gathered}
$$

of propositions of the following Lemma 1 prove Cases 1, 2, 3, 5, 7 respectively.

The Propositions $4_{*}, 6_{*}$, and $9_{*}$ of Lemma 1 imply the Propositions 4, 6 , 8-11 of Theorem 4 because Case 8 is adjacent to Case 4 and Cases 10, 11 are adjacent to Case 9 .

It remains to prove that Case 12 , namely, $\# J \geq 3$ is not $\Lambda_{1}$-simple. Indeed, the tangent plane to the first open Whitney umbrella $\Lambda_{1}$ at the point

$$
p_{1}=x_{2} t, \quad p_{2}=t^{3} / 3, \quad p_{3}=\cdots=p_{n}=0, \quad x_{1}=t^{2} / 2
$$

is defined by the equations:

$$
t d p_{1}-x_{2} d x_{1}-t^{2} d x_{2}=0, \quad d p_{2}-t d x_{1}=0, \quad d p_{3}=\cdots=d p_{n}=0 .
$$

Along the curve

$$
\begin{aligned}
& p_{1}=t^{3}, \quad p_{2}=t^{3} / 3, \quad p_{3}=\cdots=p_{n}=0, \\
& x_{1}=t^{2} / 2, \quad x_{2}=t^{2}, \quad x_{3}=\cdots=x_{n}=0
\end{aligned}
$$

our tangent plane is defined by the equations

$$
d p_{1}-t d x_{1}-t d x_{2}=0, \quad d p_{2}-t d x_{1}=0, \quad d p_{3}=\cdots=d p_{n}=0
$$

and tends to the plane $d p_{1}=\cdots=d p_{n}=0$ as $t \rightarrow 0$. Therefore, the case $\# J \geq 3$ is adjacent to the class of ordered pairs of germs of smooth Lagrange submanifolds whose tangent planes have three-dimensional intersection. This class is not simple up to symplectic equivalence because, according to $[\mathbf{1 0}]$, it corresponds to the so-called $P_{8}$ class consisting of the germs of smooth functions at critical points of corank 3. Moreover, the symplectic equivalence of ordered pairs of Lagrange germs corresponds to the stable right equivalence of the germs of smooth functions (see [10]). But the $P_{8}$ class contains a continuous invariant up to stable right equivalence [3]. This invariant comes from linear equivalence of cubic forms of three variables.

Lemma 1. Let $\alpha_{I}=\operatorname{deg} p_{I}$ and $\beta_{J}=\operatorname{deg} x_{J}$ be positive integer quasidegrees and $\mathcal{A}_{0} \supset \mathcal{A}_{1} \supset \ldots$ be the corresponding quasihomogeneous filtration in the algebra of germs at 0 of smooth functions of $p_{I}$ and $x_{J}$. Then

$\left.1_{l}\right) n=2, J=\emptyset, \alpha_{1}=\alpha_{2}=1, w_{l} \in \mathcal{A}_{l}, l \geq 2 \Rightarrow w_{l} \sim_{\Lambda_{1}} 0\left(\bmod \mathcal{A}_{l+1}\right)$;

$\left.2_{l}\right) n=2, J=\{1\}, \beta_{1}=2, \alpha_{2}=3, w_{l} \in \mathcal{A}_{l}, l \geq 4 \Rightarrow w_{l} \sim_{\Lambda_{1}} \pm e_{l}$ $\left(\bmod \mathcal{A}_{l+1}\right)$ if $w_{l} \notin\left(9 p_{2}^{2}-8 x_{1}^{3}\right)+\mathcal{A}_{l+1}$, and $w_{l} \sim_{\Lambda_{1}} 0\left(\bmod \mathcal{A}_{l+1}\right)$ if $w_{l} \in\left(9 p_{2}^{2}-8 x_{1}^{3}\right)+\mathcal{A}_{l+1}$;

$\left.2_{l}^{d}\right) n=2, J=\{1\}, \beta_{1}=2, \alpha_{2}=3, w_{d} \in \mathcal{A}_{d}, d>l \geq 4 \Rightarrow \pm e_{l}+w_{d} \sim_{\Lambda_{1}}$ $\pm e_{l}\left(\bmod \mathcal{A}_{d+1}\right)$;

$\left.3_{6}\right) n=2, J=\{2\}, \alpha_{1}=3, \beta_{2}=2, w_{6} \in \mathcal{A}_{6}, w_{6, x_{2} x_{2} x_{2}} \neq 0 \Rightarrow w_{6} \sim_{\Lambda_{1}} x_{2}^{3}$ $\left(\bmod \mathcal{A}_{7}\right)$; 
$\left.3_{6}^{d}\right) n=2, J=\{2\}, \alpha_{1}=3, \beta_{2}=2, w_{d} \in \mathcal{A}_{d}, d>6 \Rightarrow x_{2}^{3}+w_{d} \sim_{\Lambda_{1}} x_{2}^{3}$ $\left(\bmod \mathcal{A}_{d+1}\right)$

56) $n=3, J=\{3\}, \alpha_{1}=\alpha_{2}=3, \beta_{3}=2, w_{6} \in \mathcal{A}_{6}, w_{6, x_{3} x_{3} x_{3}} \neq 0 \Rightarrow$ $w_{6} \sim \Lambda_{1} x_{3}^{3}\left(\bmod \mathcal{A}_{7}\right)$

$\left.5_{6}^{d}\right) n=3, J=\{3\}, \alpha_{1}=\alpha_{2}=3, \beta_{3}=2, w_{d} \in \mathcal{A}_{d}, d>6 \Rightarrow x_{3}^{3}+w_{d} \sim_{\Lambda_{1}} x_{3}^{3}$ $\left(\bmod \mathcal{A}_{d+1}\right)$

$\left.7_{l}\right) n=2, J=\{1,2\}, \beta_{1}=\beta_{2}=1, w_{l} \in \mathcal{A}_{l}, l \geq 3 \Rightarrow w_{l} \sim_{\Lambda_{1}} \pm x_{2}^{l}$ $\left(\bmod \mathcal{A}_{l+1}\right)$ if $w_{l} \notin\left(x_{1}\right)+\mathcal{A}_{l+1}$, and $w_{l} \sim_{\Lambda_{1}} 0\left(\bmod \mathcal{A}_{l+1}\right)$ if $w_{l} \in$ $\left(x_{1}\right)+\mathcal{A}_{l+1}$

$\left.7_{l}^{d}\right) n=2, J=\{1,2\}, \beta_{1}=\beta_{2}=1, w_{d} \in \mathcal{A}_{d}, d>l \geq 3 \Rightarrow \pm x_{2}^{l}+w_{d} \sim_{\Lambda_{1}}$ $\pm x_{2}^{l}\left(\bmod \mathcal{A}_{d+1}\right)$;

$\left.4_{*}\right) n=2, J=\{2\}, \alpha_{1}=2, \beta_{2}=1 \Rightarrow \mathcal{A}_{4} / \mathcal{A}_{5}$ contains a continuous invariant with respect to $\Lambda_{1}$-equivalence;

$\left.6_{*}\right) n=3, J=\{3\}, \alpha_{1}=\alpha_{2}=2, \beta_{3}=1 \Rightarrow \mathcal{A}_{4} / \mathcal{A}_{5}$ contains a continuous invariant with respect to $\Lambda_{1}$-equivalence;

$\left.9_{*}\right) n=3, J=\{1,3\}, \beta_{1}=1, \alpha_{2}=2, \beta_{3}=1, \mathcal{A}_{3}^{\prime}=\left\{w_{3} \in \mathcal{A}_{3} \mid w_{3, p_{2} x_{3}}=\right.$ $0\} \Rightarrow \mathcal{A}_{3}^{\prime} / \mathcal{A}_{4}$ contains a continuous invariant with respect to $\Lambda_{1}$-equivalence.

\section{Hamiltonian vector fields.}

It is well-known that a vector field which preserves the symplectic structure $\omega=d p \wedge d x$ is locally defined by its Hamiltonian $H$ :

$$
\dot{x}=H_{p}, \quad \dot{p}=-H_{x} .
$$

Let $L(w)$ be the Lagrange submanifold given by a generating function $w\left(p_{I}, x_{J}\right)$ :

$$
L(w)=\left\{x_{I}=w_{p_{I}}\left(p_{I}, x_{J}\right), p_{J}=-w_{x_{J}}\left(p_{I}, x_{J}\right)\right\}
$$

and $H(w)$ denote the derivative of the generating function when the Lagrange submanifold is perturbed by the vector field with a Hamiltonian $H$.

Lemma 2. $H(w)=\left.H\right|_{L(w)}+$ const.

Proof. Indeed, $\left(p_{I}, x_{J}\right)$ are coordinates on $L(w)$ and for any two points $A, B \in L(w)$

$$
\left.w\right|_{A} ^{B}=w(B)-w(A)=\int_{A}^{B} \psi_{I}
$$

along any path on $L(w)$ joining $A$ and $B$, where $\psi_{I}=x_{I} d p_{I}-p_{J} d x_{J}$. After differentiating along our Hamiltonian vector field we get

$$
\left.\left(H(w)+w_{p_{I}} \dot{p}_{I}+w_{x_{J}} \dot{x}_{J}\right)\right|_{A} ^{B}=\int_{A}^{B} H\left(\psi_{I}\right)
$$


where $H\left(\psi_{I}\right)$ is the derivative of $\psi_{I}$ along our Hamiltonian vector field. The Cartan formula implies that

$$
\begin{aligned}
\int_{A}^{B} H\left(\psi_{I}\right) & =\int_{A}^{B} d\left(x_{I} \dot{p}_{I}-p_{J} \dot{x}_{J}\right)-\dot{p} d x+\dot{x} d p \\
& =\int_{A}^{B} d\left(x_{I} \dot{p}_{I}-p_{J} \dot{x}_{J}+H\right)=\left.\left(x_{I} \dot{p}_{I}-p_{J} \dot{x}_{J}+H\right)\right|_{A} ^{B} .
\end{aligned}
$$

Comparing the two last equalities and taking into account (2) we get $\left.H(w)\right|_{A} ^{B}=\left.H\right|_{A} ^{B}$.

Open Whitney umbrellas $\Lambda=\Lambda_{k}=f_{n, k}\left(\mathbb{R}^{n}\right)$ are real algebraic sets in $\mathbb{R}^{2 n}$. In fact, the complexification $f_{\mathbb{C}}: \mathbb{C}^{n} \rightarrow \mathbb{C}^{2 n}$ of the parametrization $f: \mathbb{R}^{n} \rightarrow \mathbb{R}^{2 n}$ is proper and one to one. Therefore $f_{\mathbb{C}}\left(\mathbb{C}^{n}\right)$ is a complex algebraic set in $\mathbb{C}^{2 n}$, and $f_{\mathbb{C}}\left(\mathbb{C}^{n}\right) \cap \mathbb{R}^{2 n}=f\left(\mathbb{R}^{n}\right)$ is a real algebraic set. However, for the explicit classification, we need, furthermore, the explicit equation of $\Lambda$.

Let $\mathcal{I}\left(\Lambda_{1}\right) \subset \mathbb{R}[p, x]$ be the ideal consisting of all polynomials which vanish on the first open Whitney umbrella $\Lambda_{1}$.

\section{Lemma 3.}

$$
\mathcal{I}\left(\Lambda_{1}\right)=\left(p_{1}^{2}-2 x_{1} x_{2}^{2}, 3 p_{1} p_{2}-4 x_{1}^{2} x_{2}, 2 p_{1} x_{1}-3 p_{2} x_{2}, 9 p_{2}^{2}-8 x_{1}^{3}, p_{3}, \ldots, p_{n}\right) .
$$

Proof. It is sufficient to prove this when $n=2$. Let $\mathcal{I}_{t}\left(\Lambda_{1}\right)=\left(t^{3} / 3-\right.$ $\left.p_{2}, t^{2} / 2-x_{1}, t x_{2}-p_{1}\right) \subset \mathbb{R}\left[t, p_{1}, p_{2}, x_{1}, x_{2}\right]$ be the ideal defining the parametric form (1) of the first open Whitney umbrella $\Lambda_{1}$ if $n=2$. Then the nine polynomials $t^{2}-2 x_{1}, t p_{1}-2 x_{1} x_{2}, 3 t p_{2}-4 x_{1}^{2}, 2 t x_{1}-3 p_{2}, t x_{2}-p_{1}, p_{1}^{2}-$ $2 x_{1} x_{2}^{2}, 3 p_{1} p_{2}-4 x_{1}^{2} x_{2}, 2 p_{1} x_{1}-3 p_{2} x_{2}, 9 p_{2}^{2}-8 x_{1}^{3}$ form a Gröbner basis of the ideal $\mathcal{I}_{t}\left(\Lambda_{1}\right)$ with respect to the lexicographic order. Hence, the four last polynomials which do not depend on $t$ generate $\mathcal{I}\left(\Lambda_{1}\right) \subset \mathbb{R}\left[p_{1}, p_{2}, x_{1}, x_{2}\right]$.

\section{Proof of Lemma 1.}

In Cases $1_{l}, 2_{l}, 2_{l}^{d}, 3_{6}, 3_{6}^{d}, 5_{6}, 5_{6}^{d}, 7_{l}, 7_{l}^{d}$ we use the standard homotopy method. Namely, let $\omega_{\tau}$ be a family of generating functions depending smoothly on a parameter $\tau$ and $H_{\tau}$ be a smooth family of Hamiltonians satisfying the homological equation

$$
H_{\tau}\left(\omega_{\tau}\right)+\partial_{\tau} \omega_{\tau} \equiv 0
$$

on a segment $[0,1]$. Besides, the corresponding Hamiltonian vector fields $v_{H_{\tau}}$ are assumed to be tangent to the first open Whitney umbrella $\Lambda_{1}$ and to preserve the origin $\left(v_{H_{\tau}}(0) \equiv 0\right)$. For the Hamiltonians $H_{\tau}$ these conditions 
mean $\left.H_{\tau}\right|_{\Lambda_{1}} \equiv 0$ and $\partial_{p} H_{\tau}(0) \equiv \partial_{x} H_{\tau}(0) \equiv 0$ respectively. So, taking into account Lemma 2 we can rewrite the homological equation as

$$
\left.H_{\tau}\right|_{L\left(\omega_{\tau}\right)}+\partial_{\tau} \omega_{\tau} \equiv 0, \quad H_{\tau} \in \mathcal{H}\left(\Lambda_{1}\right)=\mathcal{E}_{\tau} \otimes\left(\mathfrak{m}^{2} \cap \mathcal{I}^{\prime}\left(\Lambda_{1}\right)\right)
$$

where $\mathcal{E}_{\tau}$ is the algebra of smooth functions on the segment $[0,1], \mathfrak{m}$ is the maximal ideal in the algebra of germs at 0 of smooth functions on $E$, and $\mathcal{I}^{\prime}\left(\Lambda_{1}\right)$ is the ideal consisting of all germs which vanish on the first open Whitney umbrella $\Lambda_{1}$.

Now solving the Cauchy problem

$$
\dot{g}_{\tau}(p, x) \equiv v_{H_{\tau}}\left(g_{\tau}(p, x)\right), \quad g_{0}(p, x)=(p, x)
$$

with respect to a family of diffeomorphisms $g_{\tau}$ on the segment $[0,1]$ for small $(p, x)$ we get the equivalence $\omega_{0} \sim_{\Lambda_{1}} \omega_{1}$ performed by the local symplectic diffeomorphism $g_{1}$ preserving $\Lambda_{1}$ and 0 .

Let $\left.\mathcal{H}\left(\Lambda_{1}\right)\right|_{L\left(\omega_{\tau}\right)} \subset \mathcal{E}_{\tau} \otimes \mathcal{A}_{0}$ be the restriction of the ideal $\mathcal{H}\left(\Lambda_{1}\right)$ onto the family of Lagrange submanifolds given by the family $\omega_{\tau}\left(p_{I}, x_{J}\right) \in \mathcal{E}_{\tau} \otimes \mathcal{A}_{0}$ of generating functions and $\left.\operatorname{Gr} \mathcal{H}\left(\Lambda_{1}\right)\right|_{L\left(\omega_{\tau}\right)} \subset \mathcal{E}_{\tau} \otimes \mathcal{A}_{0}$ be the quasihomogeneous ideal generated by the principal quasihomogeneous parts of the germs from $\left.\mathcal{H}\left(\Lambda_{1}\right)\right|_{L\left(\omega_{\tau}\right)}$. Let us note that

$$
\left.\operatorname{Gr} \mathcal{H}\left(\Lambda_{1}\right)\right|_{L\left(\omega_{\tau}\right)} \cap\left(\mathcal{E}_{\tau} \otimes \mathcal{A}_{d}\right)=\left.\mathcal{H}\left(\Lambda_{1}\right)\right|_{L\left(\omega_{\tau}\right)} \cap\left(\mathcal{E}_{\tau} \otimes \mathcal{A}_{d}\right) \quad\left(\bmod \mathcal{E}_{\tau} \otimes \mathcal{A}_{d+1}\right) .
$$

Therefore, in the considered cases, the homological equation

$$
\left.H_{\tau}\right|_{L\left(\omega_{\tau}\right)}+\partial_{\tau} \omega_{\tau} \equiv 0\left(\bmod \mathcal{E}_{\tau} \otimes \mathcal{A}_{d+1}\right), \quad H_{\tau} \in \mathcal{H}\left(\Lambda_{1}\right)
$$

is solvable because

$$
\left.\partial_{\tau} \omega_{\tau} \in \operatorname{Gr} \mathcal{H}\left(\Lambda_{1}\right)\right|_{L\left(\omega_{\tau}\right)} \cap\left(\mathcal{E}_{\tau} \otimes \mathcal{A}_{d}\right)
$$

that is shown below in each case.

$1_{l}$ ) Let $\omega_{\tau}=\tau w_{l}$. According to Lemma 3 ,

$$
p_{1}^{2}-\left.2 x_{1} x_{2}^{2}\right|_{L\left(\omega_{\tau}\right)}, 3 p_{1} p_{2}-\left.4 x_{1}^{2} x_{2}\right|_{L\left(\omega_{\tau}\right)}, 9 p_{2}^{2}-\left.\left.8 x_{1}^{3}\right|_{L\left(\omega_{\tau}\right)} \in \mathcal{H}\left(\Lambda_{1}\right)\right|_{L\left(\omega_{\tau}\right)} .
$$

Since $L\left(\omega_{\tau}\right)=\left\{x_{1}=x_{2}=0\left(\bmod \mathcal{E}_{\tau} \otimes \mathcal{A}_{1}\right)\right\}$, the principal parts of these polynomials are $p_{1}^{2}, 3 p_{1} p_{2}, 9 p_{2}^{2}$. Therefore, $\left.\operatorname{Gr} \mathcal{H}\left(\Lambda_{1}\right)\right|_{L\left(\omega_{\tau}\right)} \supset\left(p_{1}^{2}, p_{1} p_{2}, p_{2}^{2}\right)=$ $\mathcal{E}_{\tau} \otimes \mathcal{A}_{2}$.

$\left.2_{l}\right)$ Let $w_{l}=a e_{l}+\widetilde{w}_{l}\left(\bmod \mathcal{A}_{l+1}\right)$ where $\widetilde{w}_{l}$ is a quasihomogeneous element of the ideal $\left(9 p_{2}^{2}-8 x_{1}^{3}\right)$ such that $\operatorname{deg} \widetilde{w}_{l}=l, \omega_{\tau}=a(\tau) e_{l}+\tau \widetilde{w}_{l}, a(0)=\operatorname{sign}(a)$, $a(1)=a$. According to Lemma 3 ,

$$
2 p_{1} x_{1}-\left.3 p_{2} x_{2}\right|_{L\left(\omega_{\tau}\right)}, 9 p_{2}^{2}-\left.\left.8 x_{1}^{3}\right|_{L\left(\omega_{\tau}\right)} \in \mathcal{H}\left(\Lambda_{1}\right)\right|_{L\left(\omega_{\tau}\right)} .
$$

Since $L\left(\omega_{\tau}\right)=\left\{p_{1}=-\partial_{x_{1}} \omega_{\tau}, x_{2}=\partial_{p_{2}} \omega_{\tau}\right\}$, the principal parts of this polynomials are $\mp l \omega_{\tau}, 9 p_{2}^{2}-8 x_{1}^{3}$. Therefore, $\left.\operatorname{Gr} \mathcal{H}\left(\Lambda_{1}\right)\right|_{L\left(\omega_{\tau}\right)} \supset\left(a(\tau) e_{l}, 9 p_{2}^{2}-\right.$ $\left.8 x_{1}^{3}\right) \ni \partial_{\tau} \omega_{\tau}$ if $a(\tau) \equiv 0$ or $a(\tau) \neq 0$ for $\forall \tau \in[0,1]$. 
$\left.2_{l}^{d}\right)$ Let $\omega_{\tau}= \pm e_{l}+\tau w_{d}$. According to Lemma 3,

$$
3 p_{1} p_{2}-\left.4 x_{1}^{2} x_{2}\right|_{L\left(\omega_{\tau}\right)}, 2 p_{1} x_{1}-\left.3 p_{2} x_{2}\right|_{L\left(\omega_{\tau}\right)}, 9 p_{2}^{2}-\left.\left.8 x_{1}^{3}\right|_{L\left(\omega_{\tau}\right)} \in \mathcal{H}\left(\Lambda_{1}\right)\right|_{L\left(\omega_{\tau}\right)} .
$$

Since

$$
L\left(\omega_{\tau}\right)=\left\{p_{1}=\mp \partial_{x_{1}} e_{l}\left(\bmod \mathcal{A}_{l-1}\right), x_{2}= \pm \partial_{p_{2}} e_{l}\left(\bmod \mathcal{A}_{l-2}\right)\right\},
$$

the principal parts of these polynomials are

$$
\mp\left(3 p_{2} \partial_{x_{1}} e_{l}+4 x_{1}^{2} \partial_{p_{2}} e_{l}\right), \quad \mp\left(2 x_{1} \partial_{x_{1}} e_{l}+3 p_{2} \partial_{p_{2}} e_{l}\right)=\mp l e_{l}, \quad 9 p_{2}^{2}-8 x_{1}^{3} .
$$

But

$3 p_{2} \partial_{x_{1}} e_{l}+4 x_{1}^{2} \partial_{p_{2}} e_{l}=\left\{\begin{aligned} 3 k e_{l+1} & \text { if } \quad e_{l}=x_{1}^{k} \\ (8 k / 3+4) e_{l+1}\left(\bmod \left(9 p_{2}^{2}-8 x_{1}^{3}\right)\right) & \text { if } \quad e_{l}=x_{1}^{k} p_{2}\end{aligned}\right.$ and $\left.\operatorname{Gr} \mathcal{H}\left(\Lambda_{1}\right)\right|_{L\left(\omega_{\tau}\right)} \supset\left(e_{l+1}, e_{l}, 9 p_{2}^{2}-8 x_{1}^{3}\right) \supset \mathcal{E}_{\tau} \otimes \mathcal{A}_{l}$.

$\left.3_{6}\right)$ Let $w_{6}=a p_{1}^{2}+b x_{2}^{3}\left(\bmod \mathcal{A}_{7}\right), \omega_{\tau}=a(\tau) p_{1}^{2}+b(\tau) x_{2}^{3}, a(0)=0, b(0)=$ $\operatorname{sign}(b), a(1)=a, b(1)=b$. According to Lemma 3,

$$
p_{1}^{2}-\left.2 x_{1} x_{2}^{2}\right|_{L\left(\omega_{\tau}\right)}, 2 p_{1} x_{1}-\left.\left.3 p_{2} x_{2}\right|_{L\left(\omega_{\tau}\right)} \in \mathcal{H}\left(\Lambda_{1}\right)\right|_{L\left(\omega_{\tau}\right)} .
$$

Since

$$
L\left(\omega_{\tau}\right)=\left\{x_{1}=2 a(\tau) p_{1}, p_{2}=-3 b(\tau) x_{2}^{2}\right\},
$$

the principal parts of these polynomials are $p_{1}^{2}, 4 a(\tau) p_{1}^{2}+9 b(\tau) x_{2}^{3}$. Therefore, $\left.\operatorname{Gr} \mathcal{H}\left(\Lambda_{1}\right)\right|_{L\left(\omega_{\tau}\right)} \supset\left(p_{1}^{2}, b(\tau) x_{2}^{3}\right) \ni \partial_{\tau} \omega_{\tau}$ if $b(\tau) \neq 0$ for $\forall \tau \in[0,1]$.

The change $\left(p_{2}, x_{2}\right) \mapsto-\left(p_{2}, x_{2}\right)$ shows us that $x_{2}^{3} \sim_{\Lambda_{1}}-x_{2}^{3}$.

$\left.3_{6}^{d}\right)$ Let $\omega_{\tau}=x_{2}^{3}+\tau w_{d}$. According to Lemma 3 ,

$p_{1}^{2}-\left.2 x_{1} x_{2}^{2}\right|_{L\left(\omega_{\tau}\right)}, 3 p_{1} p_{2}-\left.4 x_{1}^{2} x_{2}\right|_{L\left(\omega_{\tau}\right)}, 2 p_{1} x_{1}-\left.\left.3 p_{2} x_{2}\right|_{L\left(\omega_{\tau}\right)} \in \mathcal{H}\left(\Lambda_{1}\right)\right|_{L\left(\omega_{\tau}\right)}$.

Since $L\left(\omega_{\tau}\right)=\left\{x_{1}=0\left(\bmod \mathcal{A}_{4}\right), p_{2}=-3 x_{2}^{2}\left(\bmod \mathcal{A}_{5}\right)\right\}$, the principal parts of these polynomials are $p_{1}^{2},-9 p_{1} x_{2}^{2}, 9 x_{2}^{3}$. Therefore, $\left.\operatorname{Gr} \mathcal{H}\left(\Lambda_{1}\right)\right|_{L\left(\omega_{\tau}\right)} \supset$ $\left(p_{1}^{2}, p_{1} x_{2}^{2}, x_{2}^{3}\right)=\mathcal{E}_{\tau} \otimes \mathcal{A}_{6}$.

$\left.5_{6}\right)$ Let $w_{6}=a_{11} p_{1}^{2}+a_{12} p_{1} p_{2}+a_{22} p_{2}^{2}+b x_{3}^{3}\left(\bmod \mathcal{A}_{7}\right), \omega_{\tau}=a_{11}(\tau) p_{1}^{2}+$ $a_{12}(\tau) p_{1} p_{2}+a_{22}(\tau) p_{2}^{2}+b(\tau) x_{3}^{3}, a_{11}(0)=a_{12}(0)=a_{22}(0)=0, b(0)=\operatorname{sign}(b)$, $a_{11}(1)=a_{11}, a_{12}(1)=a_{12}, a_{22}(1)=a_{22}, b(1)=b$. According to Lemma 3 ,

$$
\begin{aligned}
& p_{1}^{2}-\left.2 x_{1} x_{2}^{2}\right|_{L\left(\omega_{\tau}\right)}, \quad 3 p_{1} p_{2}-\left.4 x_{1}^{2} x_{2}\right|_{L\left(\omega_{\tau}\right)}, \\
& \quad 9 p_{2}^{2}-\left.8 x_{1}^{3}\right|_{L\left(\omega_{\tau}\right)},\left.\left.\quad p_{3} x_{3}\right|_{L\left(\omega_{\tau}\right)} \in \mathcal{H}\left(\Lambda_{1}\right)\right|_{L\left(\omega_{\tau}\right)} .
\end{aligned}
$$

Since $L\left(\omega_{\tau}\right)=\left\{x_{1}=2 a_{11}(\tau) p_{1}+a_{12}(\tau) p_{2}, x_{2}=a_{12}(\tau) p_{1}+2 a_{22}(\tau) p_{2}, p_{3}=\right.$ $\left.-3 b(\tau) x_{3}^{2}\right\}$, the principal parts of these polynomials are $p_{1}^{2}, 3 p_{1} p_{2}, 9 p_{2}^{2}$, $-3 b(\tau) x_{3}^{3}$. Therefore, $\left.\operatorname{Gr} \mathcal{H}\left(\Lambda_{1}\right)\right|_{L\left(\omega_{\tau}\right)} \supset\left(p_{1}^{2}, p_{1} p_{2}, p_{2}^{2}, b(\tau) x_{3}^{3}\right) \ni \partial_{\tau} \omega_{\tau}$ if $b(\tau)$ $\neq 0$ for $\forall \tau \in[0,1]$. 
The change $\left(p_{3}, x_{3}\right) \mapsto-\left(p_{3}, x_{3}\right)$ shows us that $x_{3}^{3} \sim_{\Lambda_{1}}-x_{3}^{3}$. $\left.5_{6}^{d}\right)$ Let $\omega_{\tau}=x_{3}^{3}+\tau w_{d}$. According to Lemma 3,

$$
\begin{aligned}
p_{1}^{2}-\left.2 x_{1} x_{2}^{2}\right|_{L\left(\omega_{\tau}\right)}, 3 p_{1} p_{2}-\left.4 x_{1}^{2} x_{2}\right|_{L\left(\omega_{\tau}\right)}, 9 p_{2}^{2}-\left.8 x_{1}^{3}\right|_{L\left(\omega_{\tau}\right)}, \\
\left.p_{1} p_{3}\right|_{L\left(\omega_{\tau}\right)},\left.p_{2} p_{3}\right|_{L\left(\omega_{\tau}\right)},\left.\left.p_{3} x_{3}\right|_{L\left(\omega_{\tau}\right)} \in \mathcal{H}\left(\Lambda_{1}\right)\right|_{L\left(\omega_{\tau}\right)} .
\end{aligned}
$$

Since $L\left(\omega_{\tau}\right)=\left\{x_{1}=0\left(\bmod \mathcal{A}_{4}\right), x_{2}=0\left(\bmod \mathcal{A}_{4}\right), p_{3}=-3 x_{3}^{2}\left(\bmod \mathcal{A}_{5}\right)\right\}$, the principal parts of these polynomials are $p_{1}^{2}, 3 p_{1} p_{2}, 9 p_{2}^{2},-3 p_{1} x_{3}^{2},-3 p_{2} x_{3}^{2}$, $-3 x_{3}^{3}$. Therefore, $\left.\operatorname{Gr} \mathcal{H}\left(\Lambda_{1}\right)\right|_{L\left(\omega_{\tau}\right)} \supset\left(p_{1}^{2}, p_{1} p_{2}, p_{2}^{2}, p_{1} x_{3}^{2}, p_{2} x_{3}^{2}, x_{3}^{3}\right)=\mathcal{E}_{\tau} \otimes \mathcal{A}_{6}$.

$\left.7_{l}\right)$ Let $w_{l}=a x_{2}^{l}+x_{1} \widetilde{w}_{l-1}\left(\bmod \mathcal{A}_{l+1}\right)$ where $\widetilde{w}_{l-1}$ is a quasihomogeneous germ such that $\operatorname{deg} \widetilde{w}_{l-1}=l-1, \omega_{\tau}=a(\tau) x_{2}^{l}+\tau x_{1} \widetilde{w}_{l-1}, a(0)=\operatorname{sign}(a)$, $a(1)=a$. According to Lemma 3 ,

$$
\begin{aligned}
p_{1}^{2}-\left.2 x_{1} x_{2}^{2}\right|_{L\left(\omega_{\tau}\right)}, & 3 p_{1} p_{2}-\left.4 x_{1}^{2} x_{2}\right|_{L\left(\omega_{\tau}\right)}, \\
& 2 p_{1} x_{1}-\left.3 p_{2} x_{2}\right|_{L\left(\omega_{\tau}\right)}, 9 p_{2}^{2}-\left.\left.8 x_{1}^{3}\right|_{L\left(\omega_{\tau}\right)} \in \mathcal{H}\left(\Lambda_{1}\right)\right|_{L\left(\omega_{\tau}\right)} .
\end{aligned}
$$

Since $L\left(\omega_{\tau}\right)=\left\{p_{1}=-\partial_{x_{1}} \omega_{\tau}, p_{2}=-\partial_{x_{2}} \omega_{\tau}\right\}$, the principal parts of these polynomials are $-2 x_{1} x_{2}^{2},-4 x_{1}^{2} x_{2},-2 x_{1} \partial_{x_{1}} \omega_{\tau}+3 l a(\tau) x_{2}^{l}+3 \tau x_{1} x_{2} \partial_{x_{2}} \widetilde{w}_{l-1}$, $-8 x_{1}^{3}$. Therefore, $\left.\operatorname{Gr} \mathcal{H}\left(\Lambda_{1}\right)\right|_{L\left(\omega_{\tau}\right)} \supset\left(x_{1}^{3}, x_{1}^{2} x_{2}, x_{1} x_{2}^{2}, a(\tau) x_{2}^{l}\right) \ni \partial_{\tau} \omega_{\tau}$ if $a(\tau) \equiv$ 0 or $a(\tau) \neq 0$ for $\forall \tau \in[0,1]$.

$7_{l}^{d}$ ) Let $\omega_{\tau}= \pm x_{2}^{l}+\tau w_{d}$. According to Lemma 3,

$$
\begin{aligned}
p_{1}^{2}-\left.2 x_{1} x_{2}^{2}\right|_{L\left(\omega_{\tau}\right)}, & 3 p_{1} p_{2}-\left.4 x_{1}^{2} x_{2}\right|_{L\left(\omega_{\tau}\right)}, \\
& 2 p_{1} x_{1}-\left.3 p_{2} x_{2}\right|_{L\left(\omega_{\tau}\right)}, 9 p_{2}^{2}-\left.\left.8 x_{1}^{3}\right|_{L\left(\omega_{\tau}\right)} \in \mathcal{H}\left(\Lambda_{1}\right)\right|_{L\left(\omega_{\tau}\right)} .
\end{aligned}
$$

Since $L\left(\omega_{\tau}\right)=\left\{p_{1}=0\left(\bmod \mathcal{A}_{l}\right), p_{2}=\mp l x_{2}^{l-1}\left(\bmod \mathcal{A}_{l}\right)\right\}$, the principal parts of these polynomials are $-2 x_{1} x_{2}^{2},-4 x_{1}^{2} x_{2}, \pm 3 l x_{2}^{l},-8 x_{1}^{3}$. Therefore,

$$
\left.\operatorname{Gr} \mathcal{H}\left(\Lambda_{1}\right)\right|_{L\left(\omega_{\tau}\right)} \supset\left(x_{1}^{3}, x_{1}^{2} x_{2}, x_{1} x_{2}^{2}, x_{2}^{l}\right) \supset \mathcal{E}_{\tau} \otimes \mathcal{A}_{l} .
$$

In Cases $4_{*}, 6_{*}$, and $9_{*}$ we consider the Lie algebra of germs of Hamiltonian vector fields which are tangent to the first open Whitney umbrella $\Lambda_{1}$ and preserve 0. For the Hamiltonians $H$ these conditions mean $\left.H\right|_{\Lambda_{1}}=0$ and $\partial_{p} H(0)=\partial_{x} H(0)=0$ respectively. So, our Lie algebra is the ideal $\mathfrak{m}^{2} \cap$ $\mathcal{I}^{\prime}\left(\Lambda_{1}\right)$ where $\mathfrak{m}$ is the maximal ideal in the algebra of germs at 0 of smooth functions on $E$ and $\mathcal{I}^{\prime}\left(\Lambda_{1}\right)$ is the ideal consisting of all germs which vanish on the first open Whitney umbrella $\Lambda_{1}$. 
Let $\mathcal{B}=\mathcal{A}_{4} / \mathcal{A}_{5}$ in Cases $4_{*}, 6_{*}$ and $\mathcal{B}=\mathcal{A}_{3}^{\prime} / \mathcal{A}_{4}$ in Case $9_{*}$. In all these cases our Lie algebra $\mathfrak{m}^{2} \cap \mathcal{I}^{\prime}\left(\Lambda_{1}\right)$ acts on $\mathcal{B}$ by the formula from Lemma 2 : $H(\omega)=\left.H\right|_{L(\omega)}$. But it turns out that, for any $\omega \in \mathcal{B}$,

$$
\left.\operatorname{dim} \mathfrak{m}^{2} \cap \mathcal{I}^{\prime}\left(\Lambda_{1}\right)\right|_{L(\omega)}<\operatorname{dim} \mathcal{B} .
$$

It remains to check these inequalities.

$4_{*}$ ) Let $\omega=a p_{1}^{2}+b p_{1} x_{2}^{2}+c x_{2}^{4} \in \mathcal{B}$ where $\mathcal{B}=\mathcal{A}_{4} / \mathcal{A}_{5}$. Then

$$
\begin{gathered}
L(\omega)=\left\{x_{1}=2 a p_{1}+b x_{2}^{2}, p_{2}=-2 b p_{1} x_{2}-4 c x_{2}^{3}\right\}, \\
p_{1}^{2}-\left.2 x_{1} x_{2}^{2}\right|_{L(\omega)} \in \mathcal{B}, \quad 2 p_{1} x_{1}-\left.3 p_{2} x_{2}\right|_{L(\omega)} \in \mathcal{B}, \\
3 p_{1} p_{2}-\left.4 x_{1}^{2} x_{2}\right|_{L(\omega)}=9 p_{2}^{2}-\left.8 x_{1}^{3}\right|_{L(\omega)}=0\left(\bmod \mathcal{A}_{5}\right) .
\end{gathered}
$$

Therefore, according to Lemma 3,

$$
\left.\operatorname{dim} \mathfrak{m}^{2} \cap \mathcal{I}^{\prime}\left(\Lambda_{1}\right)\right|_{L(\omega)} \leq 2
$$

but $\operatorname{dim} \mathcal{B}=3$.

$\left.6_{*}\right)$ Let $\omega=a_{1} p_{1}^{2}+a_{2} p_{1} p_{2}+a_{3} p_{2}^{2}+b_{1} p_{1} x_{3}^{2}+b_{2} p_{2} x_{3}^{2}+c x_{3}^{4} \in \mathcal{B}$ where $\mathcal{B}=$ $\mathcal{A}_{4} / \mathcal{A}_{5}$. Then

$$
\begin{aligned}
L(\omega)=\left\{x_{1}=2 a_{1} p_{1}+a_{2} p_{2}+b_{1} x_{3}^{2}, x_{2}\right. & =a_{2} p_{1}+2 a_{3} p_{2}+b_{2} x_{3}^{2}, \\
p_{3} & \left.=-2 b_{1} p_{1} x_{3}-2 b_{2} p_{2} x_{3}-4 c x_{2}^{3}\right\},
\end{aligned}
$$

$$
p_{1}^{2}-\left.2 x_{1} x_{2}^{2}\right|_{L(\omega)}=p_{1}^{2} \quad\left(\bmod \mathcal{A}_{5}\right) \in \mathcal{B}
$$

$$
3 p_{1} p_{2}-\left.4 x_{1}^{2} x_{2}\right|_{L(\omega)}=3 p_{1} p_{2} \quad\left(\bmod \mathcal{A}_{5}\right) \in \mathcal{B},
$$

$2 p_{1} x_{1}-\left.3 p_{2} x_{2}\right|_{L(\omega)} \in \mathcal{B}, 9 p_{2}^{2}-\left.8 x_{1}^{3}\right|_{L(\omega)}=9 p_{2}^{2} \quad\left(\bmod \mathcal{A}_{5}\right) \in \mathcal{B},\left.\quad p_{3} x_{3}\right|_{L(\omega)} \in \mathcal{B}$,

$$
\left.p_{1} p_{3}\right|_{L(\omega)}=\left.p_{2} p_{3}\right|_{L(\omega)}=\left.p_{3}^{2}\right|_{L(\omega)}=\left.x_{1} p_{3}\right|_{L(\omega)}=\left.x_{2} p_{3}\right|_{L(\omega)}=0\left(\bmod \mathcal{A}_{5}\right) .
$$

Therefore, according to Lemma 3,

$$
\left.\operatorname{dim} \mathfrak{m}^{2} \cap \mathcal{I}^{\prime}\left(\Lambda_{1}\right)\right|_{L(\omega)} \leq 5
$$

but $\operatorname{dim} \mathcal{B}=6$.

$\left.9_{*}\right)$ Let $\omega=a x_{1} p_{2}+b_{1} x_{1}^{3}+b_{2} x_{1}^{2} x_{3}+b_{3} x_{1} x_{3}^{2}+b_{4} x_{3}^{3} \in \mathcal{B}$ where $\mathcal{B}=\mathcal{A}_{3}^{\prime} / \mathcal{A}_{4}$. Then

$$
\begin{array}{r}
L(\omega)=\left\{p_{1}=-a p_{2}-3 b_{1} x_{1}^{2}-2 b_{2} x_{1} x_{3}-b_{3} x_{3}^{2}, x_{2}=a x_{1},\right. \\
\left.p_{3}=-b_{2} x_{1}^{2}-2 b_{3} x_{1} x_{3}-3 b_{4} x_{3}^{2}\right\},
\end{array}
$$




$$
\begin{gathered}
p_{1}^{2}-\left.2 x_{1} x_{2}^{2}\right|_{L(\omega)}=-2 a^{2} x_{1}^{3} \quad\left(\bmod \mathcal{A}_{4}\right) \in \mathcal{B}, \\
3 p_{1} p_{2}-\left.4 x_{1}^{2} x_{2}\right|_{L(\omega)}=-4 a x_{1}^{3} \quad\left(\bmod \mathcal{A}_{4}\right) \in \mathcal{B}, \\
2 p_{1} x_{1}-\left.3 p_{2} x_{2}\right|_{L(\omega)}=-5 a x_{1} p_{2}-6 b_{1} x_{1}^{3}-4 b_{2} x_{1}^{2} x_{3}-2 b_{3} x_{1} x_{3}^{2} \in \mathcal{B}, \\
9 p_{2}^{2}-\left.8 x_{1}^{3}\right|_{L(\omega)}=-8 x_{1}^{3}\left(\bmod \mathcal{A}_{4}\right) \in \mathcal{B}, \\
\left.x_{1} p_{3}\right|_{L(\omega)}=-b_{2} x_{1}^{3}-2 b_{3} x_{1}^{2} x_{3}-3 b_{4} x_{1} x_{3}^{2} \in \mathcal{B},\left.\quad x_{2} p_{3}\right|_{L(\omega)}=\left.a x_{1} p_{3}\right|_{L(\omega)} \in \mathcal{B}, \\
\left.p_{3} x_{3}\right|_{L(\omega)}=-b_{2} x_{1}^{2} x_{3}-2 b_{3} x_{1} x_{3}^{2}-3 b_{4} x_{3}^{3} \in \mathcal{B}, \\
\left.p_{1} p_{3}\right|_{L(\omega)}=\left.p_{2} p_{3}\right|_{L(\omega)}=\left.p_{3}^{2}\right|_{L(\omega)}=0 \quad\left(\bmod \mathcal{A}_{4}\right) .
\end{gathered}
$$

Therefore, according to Lemma 3,

$$
\left.\operatorname{dim} \mathfrak{m}^{2} \cap \mathcal{I}^{\prime}\left(\Lambda_{1}\right)\right|_{L(\omega)} \leq 4
$$

but $\operatorname{dim} \mathcal{B}=5$.

\section{Stable Lagrange mappings.}

In this Section we prove Theorems 1 and 2. Theorem 1 follows from Theorem 2, the proved Theorem 3, and the following Lemma 4.

Definition. Let $\Lambda \subset E$ be a Lagrange variety. The germ at 0 of the Lagrange fibration given by a generating family $W\left(p_{I}, x_{J}, y\right)$ such that $w\left(p_{I}, x_{J}\right)$ $=W\left(p_{I}, x_{J}, 0\right)$ is called $\Lambda$-versal if

$$
\left.\mathcal{I}^{\prime}(\Lambda)\right|_{L(w)}+\left\langle\left. W_{y}\right|_{y=0}, 1\right\rangle_{\mathbb{R}}=\mathcal{A}_{0}
$$

where $\mathcal{I}^{\prime}(\Lambda)$ is the ideal consisting of the germs of all functions on $E$ which vanish on the Lagrange variety $\Lambda, L(w) \subset E$ is the Lagrange submanifold defined by the generating function $w$, and $\mathcal{A}_{0}$ is the algebra of germs at 0 of smooth functions of $p_{I}$ and $x_{J}$.

Remark. This is nothing but the Givental' versality [9] for the Lagrange mapping $\Lambda \subset E \rightarrow Y$ when the Lagrange fibration is defined by the generating family $W$. Also, in the case $\Lambda$ is an open Whitney umbrella, the Givental' versality condition is equivalent to that the parametrization of $\Lambda$ is Lagrange stable with respect to the Lagrange fibration in the sense of [13], [15] (Theorem 2 from [13], page 216).

Lemma 4. The germs of Lagrange fibrations from Theorem 1 are stable with respect to $\Lambda_{1}$-equivalence. 
Proof. Let $\alpha_{I}=\operatorname{deg} p_{I}$ and $\beta_{J}=\operatorname{deg} x_{J}$ be positive integer quasidegrees, $\mathcal{A}_{0} \supset \mathcal{A}_{1} \supset \ldots$ the corresponding quasihomogeneous filtration in the algebra of germs at 0 of smooth functions of $p_{I}$ and $x_{J}$, and $\left.\operatorname{Gr} \mathcal{I}^{\prime}\left(\Lambda_{1}\right)\right|_{L\left(\omega_{\tau}\right)} \subset \mathcal{A}_{0}$ the quasihomogeneous ideal generated by the principal quasihomogeneous parts of the germs from $\left.\mathcal{I}^{\prime}\left(\Lambda_{1}\right)\right|_{L\left(\omega_{\tau}\right)}$. Let us note that

$$
\left.\operatorname{Gr} \mathcal{I}^{\prime}\left(\Lambda_{1}\right)\right|_{L\left(\omega_{\tau}\right)} \cap \mathcal{A}_{d}=\left.\mathcal{I}^{\prime}\left(\Lambda_{1}\right)\right|_{L\left(\omega_{\tau}\right)} \cap \mathcal{A}_{d}\left(\bmod \mathcal{A}_{d+1}\right) .
$$

By analogy with the cases $1_{l}, 2_{l}^{d}, 3_{6}^{d}, 7_{l}^{d}, 5_{6}^{d}$ from the proof of Lemma 1 we get

$\left.S_{3}\right) w\left(p_{1}, p_{2}\right)=0, \alpha_{1}=\alpha_{2}=\left.1 \Rightarrow \operatorname{Gr} \mathcal{I}^{\prime}\left(\Lambda_{1}\right)\right|_{L(w)} \supset\left(p_{1}^{2}, p_{1} p_{2}, p_{2}^{2}\right)=\mathcal{A}_{2} ;$

$\left.S_{m}^{ \pm}\right) w\left(x_{1}, p_{2}\right)= \pm e_{m+1}, \beta_{1}=2, \alpha_{2}=\left.3 \Rightarrow \operatorname{Gr} \mathcal{I}^{\prime}\left(\Lambda_{1}\right)\right|_{L(w)} \supset\left(e_{m+1}, e_{m+2}\right.$, $\left.9 p_{2}^{2}-8 x_{1}^{3}\right) \supset \mathcal{A}_{m+1}$

T5) $w\left(p_{1}, x_{2}\right)=x_{2}^{3}, \beta_{1}=3, \alpha_{2}=\left.2 \Rightarrow \operatorname{Gr} \mathcal{I}^{\prime}\left(\Lambda_{1}\right)\right|_{L(w)} \supset\left(p_{1}^{2}, p_{1} x_{2}^{2}, x_{2}^{3}\right)=\mathcal{A}_{6}$;

$\left.U_{6}\right) w\left(x_{1}, x_{2}\right)= \pm x_{2}^{m-3}, \beta_{1}=\beta_{2}=\left.1 \Rightarrow \operatorname{Gr} \mathcal{I}^{\prime}\left(\Lambda_{1}\right)\right|_{L(w)} \supset\left(x_{1}^{3}, x_{1}^{2} x_{2}, x_{1} x_{2}^{2}\right.$, $\left.x_{2}^{m-3}\right) \supset \mathcal{A}_{m-3}$

$\left.V_{6}\right) w\left(p_{1}, p_{2}, x_{3}\right)=x_{3}^{3}, \alpha_{1}=\alpha_{2}=3, \beta_{3}=\left.2 \Rightarrow \operatorname{Gr} \mathcal{I}^{\prime}\left(\Lambda_{1}\right)\right|_{L(w)} \supset\left(p_{1}^{2}, p_{1} p_{2}\right.$, $\left.p_{2}^{2}, x_{3}^{2}\right) \supset \mathcal{A}_{6}$.

Therefore, the Nakayama lemma implies that

$\left.S_{3}\right)\left.\mathcal{I}^{\prime}\left(\Lambda_{1}\right)\right|_{L(w)} \supset\left(p_{1}^{2}, p_{1} p_{2}, p_{2}^{2}\right) ;$

$\left.S_{m}^{ \pm}\right)\left.\mathcal{I}^{\prime}\left(\Lambda_{1}\right)\right|_{L(w)} \supset\left(e_{m+1}, e_{m+2}, 9 p_{2}^{2}-8 x_{1}^{3}\right)$;

$\left.T_{5}\right)\left.\mathcal{I}^{\prime}\left(\Lambda_{1}\right)\right|_{L(w)} \supset\left(p_{1}^{2}, p_{1} x_{2}^{2}, x_{2}^{3}\right)$;

$\left.U_{6}\right)\left.\mathcal{I}^{\prime}\left(\Lambda_{1}\right)\right|_{L(w)} \supset\left(x_{1}^{3}, x_{1}^{2} x_{2}, x_{1} x_{2}^{2}, x_{2}^{m-3}\right)$;

$\left.V_{6}\right)\left.\mathcal{I}^{\prime}\left(\Lambda_{1}\right)\right|_{L(w)} \supset\left(p_{1}^{2}, p_{1} p_{2}, p_{2}^{2}, x_{3}^{2}\right)$.

Hence, in the case of an arbitrary $n$ :

$\left.S_{3}\right)\left.\mathcal{I}^{\prime}\left(\Lambda_{1}\right)\right|_{L(w)} \supset\left(p_{1}^{2}, p_{1} p_{2}, p_{2}^{2}, p_{3}, \ldots, p_{n}\right) ;$

$\left.S_{m}^{ \pm}\right)\left.\mathcal{I}^{\prime}\left(\Lambda_{1}\right)\right|_{L(w)} \supset\left(e_{m+1}, e_{m+2}, 9 p_{2}^{2}-8 x_{1}^{3}, p_{3}, \ldots, p_{n}\right)$;

$\left.T_{5}\right)\left.\mathcal{I}^{\prime}\left(\Lambda_{1}\right)\right|_{L(w)} \supset\left(p_{1}^{2}, p_{1} x_{2}^{2}, x_{2}^{3}, p_{3}, \ldots, p_{n}\right)$

$\left.U_{6}\right)\left.\mathcal{I}^{\prime}\left(\Lambda_{1}\right)\right|_{L(w)} \supset\left(x_{1}^{3}, x_{1}^{2} x_{2}, x_{1} x_{2}^{2}, x_{2}^{m-3}, p_{3}, \ldots, p_{n}\right)$;

$\left.V_{6}\right)\left.\mathcal{I}^{\prime}\left(\Lambda_{1}\right)\right|_{L(w)} \supset\left(p_{1}^{2}, p_{1} p_{2}, p_{2}^{2}, x_{3}^{2}, p_{4}, \ldots, p_{n}\right)$.

So, our germs of Lagrange fibrations are $\Lambda_{1}$-versal. According to Theorem 3 from [9], they are stable with respect to $\Lambda_{1}$-equivalence.

Proof of Theorem 1. The classification at regular points is just a rewrite of Arnold's theorem. The classification at singular points follows from Theorem 3, Lemma 4, and Theorem 2.

Now we are going to prove Theorem 2 .

Lemma 5. Let $r$ be a nonnegative integer or $r=\infty$. Let $i:\left(\mathbb{R}^{n}, 0\right) \rightarrow$ $(E, 0), i^{\prime}:\left(\mathbb{R}^{n}, 0\right) \rightarrow(E, 0)$ be germs of Lagrange immersions with $j^{r} i(0)=$ 
$j^{r} i^{\prime}(0)$. Then there exists a germ of symplectic diffeomorphism $\tau$ such that $i^{\prime}=\tau \circ i$, and that $j^{r} \tau(0)=j^{r} \operatorname{id}_{E}(0)$.

Proof. It is sufficient to show in the case $i$ is the inclusion of the zerosection $\mathbb{R}^{n} \rightarrow T^{*} \mathbb{R}^{n}$ and $i^{\prime}: \mathbb{R}^{n} \rightarrow T^{*} \mathbb{R}^{n}$ is defined as the graph of $d h$ for a function $h: \mathbb{R}^{n} \rightarrow \mathbb{R}$ with $\operatorname{ord}_{0} h>r+1$. Then $\tau$ may be defined by $(p, x) \mapsto(p+d h(x), x)$.

For a germ of symplectic manifold $(E, 0)$ and a germ of Lagrange submanifold $(L, 0) \subset(E, 0)$ at a base point 0 , we denote by $\operatorname{Sp}(E, L)$ the group consisting of germs of symplectic diffeomorphisms $(E, 0) \rightarrow(E, 0)$ preserving $L$. Take a Lagrange fibration $\pi:(E, 0) \rightarrow(Y, 0)$ having $L$ as the central fiber: $\pi^{-1}(0)=L$. We denote by $\operatorname{Lag}(E, \pi)$ the group consisting of $\pi$-fiber preserving symplectic diffeomorphism-germs $(E, 0) \rightarrow(E, 0)$. Notice that $\operatorname{Lag}(E, \pi) \subset \operatorname{Sp}(E, L)$.

Lemma 6. $\operatorname{Lag}(E, \pi)$ is a deformation retract of $\operatorname{Sp}(E, L)$. More exactly, there exists a mapping $D: \operatorname{Sp}(E, L) \times[0,1] \rightarrow \operatorname{Sp}(E, L)$ with the properties:

(1) $D(\tau, 0)=\tau, D(\tau, 1) \in \operatorname{Lag}(E, \pi),(\tau \in \operatorname{Sp}(E, L))$.

(2) $D(\tau, t)=\tau,(\tau \in \operatorname{Lag}(E, \pi), t \in[0,1])$.

(3) $D(\tau, \cdot): E \times[0,1] \rightarrow E$ is smooth on $E \times[0,1]$ for each $\tau \in \operatorname{Sp}(E, L)$ and continuous on a compact neighborhood of $0 \times[0,1]$ in $E \times[0,1]$ with respect to $C^{\infty}$-topology, when $\tau$ is considered as a variable.

(4) $j^{1}\left(\left.D(\tau, t)\right|_{L}\right)(0)=j^{1} \mathrm{id}_{L}(0),(\tau \in \operatorname{Sp}(E, L), 0 \leq t \leq 1)$.

In particular, each element of $\operatorname{Sp}(E, L)$ is connected to an element of $\operatorname{Lag}(E, \pi)$ by a smooth path, fixing the 1-jet of the restriction to $L$, within $\operatorname{Sp}(E, L)$.

Proof. It suffices to show when $E=T^{*} \mathbb{R}^{n}$ with the canonical coordinates $(p, x), L=\{x=0\}$ and $\pi: T^{*} \mathbb{R}^{n} \rightarrow Y=\mathbb{R}^{n}$ is the standard projection $\pi(p, x)=x$. Let $\tau \in \operatorname{Sp}(E, L)$. Set $\tau(p, x)=(P(p, x), X(p, x))$. Then $X(p, 0)=0$. Remark that the Jacobi matrix $A=(\partial X / \partial x)(0,0)$ is regular. Now consider the graph $\Gamma(\tau)$ of $\tau$ in $T^{*} \mathbb{R}^{n} \times T^{*} \mathbb{R}^{n}$, with coordinates $=$ $\left(p, x ; p^{\prime}, x^{\prime}\right)$. Then $\Gamma(\tau)$ is a Lagrange submanifold with respect to the symplectic form $\Omega=\sum_{i} d p_{i}^{\prime} \wedge d x_{i}^{\prime}-\sum_{i} d p_{i} \wedge d x_{i}$ of $T^{*} \mathbb{R}^{n} \times T^{*} \mathbb{R}^{n}$. Consider the Lagrange projection $\Pi: T^{*} \mathbb{R}^{n} \times T^{*} \mathbb{R}^{n} \rightarrow \mathbb{R}^{2 n}$ defined by $\Pi\left(p, x, p^{\prime}, x^{\prime}\right)=\left(p, x^{\prime}\right)$. Then $\left.\Pi\right|_{\Gamma(\tau)}: \Gamma(\tau) \rightarrow \mathbb{R}^{2 n}$ is a germ of diffeomorphism at 0 . Also consider the projection $\Pi^{\prime}: T^{*} \mathbb{R}^{n} \times T^{*} \mathbb{R}^{n} \rightarrow T^{*} \mathbb{R}^{n}$ defined by $\Pi^{\prime}\left(p, x ; p^{\prime}, x^{\prime}\right)=(p, x)$, then $\Phi(\tau)=\Pi^{\prime} \circ\left(\left.\Pi\right|_{\Gamma(\tau)}\right)^{-1}: \mathbb{R}^{2 n} \rightarrow T^{*} \mathbb{R}^{n}$ is a germ of diffeomorphism at 0 . If we set $\Phi\left(p, x^{\prime}\right)=\left(p, x\left(p, x^{\prime}\right)\right)$, then the condition that $\tau$ preserves $L$ is interpreted to the equation $x(p, 0)=0$. Moreover $\tau \in \operatorname{Lag}(E, \pi)$ if and only if $x\left(p, x^{\prime}\right)$ does not depend on $p$. Remark that $\left(\frac{\partial x}{\partial x^{\prime}}\right)(0,0)$ is equal to the inverse matrix of $A$. 
Now take the generating function $H=H\left(p, x^{\prime}\right)$ of $\Gamma(\tau)$ for the 1-form $\theta=$ $\sum_{i} p_{i}^{\prime} d x_{i}^{\prime}+\sum_{i} x_{i} d p_{i}$ : We have $\left(\left.\Pi\right|_{\Gamma(\tau)}\right)^{*}(d H)=\left.\theta\right|_{\Gamma(\tau)}$, and $x=\partial H / \partial p, p^{\prime}=$ $\partial H / \partial x^{\prime}$. Then $(\partial H / \partial p)(p, 0)=0$. Now we write $H\left(p, x^{\prime}\right)=h_{0}\left(x^{\prime}\right)+$ $\sum_{i=1}^{n} h_{i}\left(x^{\prime}\right) p_{i}+I\left(p, x^{\prime}\right), I\left(p, x^{\prime}\right)$ being of order $\geq 2$ for $p$. Then $h_{i}(0)=0,1 \leq$ $i \leq n$ and $(\partial I / \partial p)(p, 0)=0$. Moreover $\tau$ belongs to $\operatorname{Lag}(E, \pi)$ if and only if the generating function $H=H\left(p, x^{\prime}\right)$ of $\Gamma(\tau)$ is an inhomogeneous linear function with respect to $p$, namely if $I\left(p, x^{\prime}\right)=0$. Then we set $H_{t}\left(p, x^{\prime}\right)=$ $h_{0}\left(x^{\prime}\right)+\sum_{i=1}^{n} h_{i}\left(x^{\prime}\right) p_{i}+(1-t) I\left(p, x^{\prime}\right), 0 \leq t \leq 1$. The restriction of $\Pi^{\prime}$ to the graph of $d H_{t}$ in $T^{*} \mathbb{R}^{n} \times T^{*} \mathbb{R}^{n}$ is a diffeomorphism, and therefore $d H_{t}$ defines a family of symplectic diffeomorphisms $\tau_{t}:\left(T^{*} \mathbb{R}^{n}, 0\right) \rightarrow\left(T^{*} \mathbb{R}^{n}, 0\right)$ in $\operatorname{Sp}(E, L)$. Then we set $D(\tau, t)=\tau_{t}$. The points (1), (2), (3) and (4) are clear.

Now we set

$$
\begin{aligned}
& \mathcal{F}:=\left\{f:\left(\mathbb{R}^{n}, 0\right) \rightarrow\left(T^{*} \mathbb{R}^{n}, 0\right) \mid f \text { is isotropic of corank } \leq 1\right\}, \\
& \operatorname{Sp}-\mathcal{A}:=\left\{(\sigma, \tau) \mid \sigma \in \operatorname{Diff}\left(\mathbb{R}^{n}, 0\right), \tau \in \operatorname{Sp}\left(T^{*} \mathbb{R}^{n}, 0\right)\right\}, \\
& \operatorname{Lag}-\mathcal{A}:=\left\{(\sigma, \tau) \mid \sigma \in \operatorname{Diff}\left(\mathbb{R}^{n}, 0\right), \tau \in \operatorname{Lag}\left(T^{*} \mathbb{R}^{n}, \pi\right)\right\} \\
&=\operatorname{Diff}\left(\mathbb{R}^{n}, 0\right) \times \operatorname{Lag}\left(T^{*} \mathbb{R}^{n}, \pi\right),
\end{aligned}
$$

group of ordinary Lagrange equivalence with respect to the canonical fibration $\pi: T^{*} \mathbb{R}^{n} \rightarrow \mathbb{R}^{n}, \pi(p, x)=x$, and set

$$
\operatorname{Lag}-\mathcal{K}:=\{(\sigma, \tau) \in \operatorname{Sp}-\mathcal{A} \mid \tau(L)=L\}=\operatorname{Diff}\left(\mathbb{R}^{n}, 0\right) \times \operatorname{Sp}\left(T^{*} \mathbb{R}^{n}, L\right),
$$

where $L=\pi^{-1}(0)$, the central fiber. Then the group Lag- $\mathcal{A}$ (resp. Lag- $\mathcal{K}$ ) acts on $\mathcal{F}$ naturally: $(\sigma, \tau) f:=\tau \circ f \circ \sigma^{-1}$. Moreover we recall that $J_{I}^{r}(n, 2 n):=\left\{j^{r} f(0) \mid f \in \mathcal{F}\right\}$ is a submanifold of the ordinary jet space $J^{r}(n, 2 n)[\mathbf{1 2}]$. Then Lag- $\mathcal{A}$ (resp. Lag- $\left.\mathcal{K}\right)$ acts on $J_{I}^{r}(n, 2 n)$ naturally as well.

Lemma 7. Let $f, f^{\prime} \in \mathcal{F}$. If $f, f^{\prime}$ are Lagrange stable and Lag- $\mathcal{K}$-equivalent, then they are Lag- $\mathcal{A}$-equivalent.

Proof. Since $f, f^{\prime}$ are Lag- $\mathcal{K}$-equivalent, there is a $(\sigma, \tau) \in$ Lag- $\mathcal{K}$ with $f^{\prime}=$ $\tau \circ f \circ \sigma^{-1}$. By Lemma 6, there is a smooth path $\tau_{t} \in \operatorname{Sp}(E, L), 0 \leq t \leq 1$, with $\tau_{0}=\tau, \tau_{1} \in \operatorname{Lag}(E, \pi)$ and $j^{1}\left(\left.\tau_{t}\right|_{L}\right)(0)=j^{1}\left(\left.\mathrm{id}\right|_{L}\right)(0)$. We set $f_{t}:=\tau_{t} \circ f$. Then we have $f_{0}=f^{\prime} \circ \sigma$.

Remark that $f_{t}, 0 \leq t \leq 1$ are Lagrange stable $([\mathbf{1 3}]$, Theorem 1.2, [15], Theorem 1.1). Therefore $f_{t},(0 \leq t \leq 1)$ are finitely Lag- $\mathcal{A}$-determined ([15], Theorem 1.3), so we may discuss on an isotropic jet space $J_{I}^{r}(n, 2 n)$ of sufficiently high order. (This argument is analogous to the ordinary one. See $[\mathbf{2 1}]$.)

The vector field $v=\left.\frac{\partial f_{t}}{\partial t}\right|_{t=t_{0}}:\left(\mathbb{R}^{n}, 0\right) \rightarrow T\left(T^{*} \mathbb{R}^{n}\right)$ along $f_{t_{0}}$ belongs to the tangent space $t f_{0}\left(m_{n} V_{n}\right)+w f_{0}\left(m_{n} V I_{2 n}\right)$ at $f_{t_{0}}$ to the Lag- $\mathcal{A}$-orbit, 
for each $t_{0} \in[0,1]$. (See [13], [15] for the notations.) In fact there exists $\eta \in m_{n} V I_{2 n}$ such that $v-\eta \circ f_{t_{0}}$ has null generating function. So we have $\xi \in V_{n}$ with $t f(\xi)=v-\eta \circ f_{t_{0}}$ (cf. [13], Lemma 4.3). Then $\xi$ is tangent to the stratum through 0 , with respect to the stratification of $\mathbb{R}^{n}$, by the types of open Whitney umbrellas. Since $t f(\xi)$ must vanish at 0 , and $f$ is immersive along each stratum, we see $v$ vanishes at 0 . Thus, by Mather's Lemma ([17] IV, pp. 534-535), we see that $j^{r} f_{t}(0),(0 \leq t \leq 1)$ belong to the single Lag$\mathcal{A}$-orbit in $J_{I}^{r}(n, 2 n)$. In particular $j^{r} f_{0}(0)$ and $j^{r} f_{1}(0)$ belong to the same Lag- $\mathcal{A}$ orbit. By the determinacy, we have $f_{0}$ and $f_{1}$ are Lag- $\mathcal{A}$-equivalent. Since $f_{0}$ and $f^{\prime}$ are Lag- $\mathcal{A}$-equivalent, and $f_{1}$ and $f$ are Lag- $\mathcal{A}$-equivalent, we see that $f$ and $f^{\prime}$ are Lag- $\mathcal{A}$-equivalent.

Proof of Theorem 2. Let $\pi, \pi^{\prime}:(E, 0) \rightarrow(Y, 0)$ be $\Lambda$-stable Lagrange projections. Set $L=\pi^{-1}(0)$ and $L^{\prime}=\pi^{\prime-1}(0)$, and assume $L$ and $L^{\prime}$ are formally $\Lambda$-equivalent. By taking symplectic coordinates, we may assume $E=T^{*} \mathbb{R}^{n}$ and $\pi: T^{*} \mathbb{R}^{n} \rightarrow \mathbb{R}^{n}$ is the standard fibration.

Then $j^{\infty} i(0)=j^{\infty}\left(\tau \circ i^{\prime}\right)(0)$, for some parametrizations $i$ of $L$ and $i^{\prime}$ of $L^{\prime}$ and for a symplectic diffeomorphism $\tau$ preserving $\Lambda$. By Lemma 5, there exists a symplectic diffeomorphism $\tau^{\prime}$ with $j^{\infty} \tau^{\prime}(0)=j^{\infty} \mathrm{id}(0)$ and $i=\tau^{\prime} \circ \tau \circ i^{\prime}$. Remark that $\tau^{\prime}$ needs not preserve $\Lambda$. Set $f^{\prime}=\tau^{\prime} \circ f$ for a parametrization (open Whitney umbrella) $f:\left(\mathbb{R}^{n}, 0\right) \rightarrow(E, 0)$ of $\Lambda$. Then $f^{\prime}$ is symplectically equivalent to $f[\mathbf{1 3}]$, and $j^{\infty} f^{\prime}(0)=j^{\infty} f(0)$. Moreover $(f, \pi)$ is Lagrange stable (Theorem 1.2 of $[\mathbf{1 3}]$ ). Then as shown in [15] Theorem 1.3, $f$ is finitely Lag- $\mathcal{A}$-determined, and therefore $\left(f^{\prime}, \pi\right)$ and $(f, \pi)$ are Lagrange equivalent by $\left(\sigma, \tau^{\prime \prime}\right)$, namely, $\tau^{\prime \prime} \circ f^{\prime}=f \circ \sigma$ and $\tau^{\prime \prime} \in \operatorname{Lag}(E, \pi)$. Set $\tau_{1}=\tau^{\prime \prime} \circ \tau^{\prime} \circ \tau$. Then $\tau_{1}(\Lambda)=\Lambda$ and $\tau_{1}\left(L^{\prime}\right)=L$. Therefore $L$ and $L^{\prime}$ are $\Lambda$-equivalent.

Now take a symplectic diffeomorphism $\tau_{2}:(E, 0) \rightarrow(E, 0)$ such that $\pi=\pi^{\prime} \circ \tau_{2}[\mathbf{3}]$. Set $\tilde{f}=\tau_{2}^{-1} \circ f$. Since $\pi$ is $\Lambda$-stable, we see that $f$ is Lagrange stable with respect to $\pi$. So is $\tilde{f}$, since $\pi^{\prime}$ is $\Lambda$-stable. Since $\tau_{1} \circ \tau_{2}$ maps $L$ to $L$ and $f\left(\mathbb{R}^{n}\right)$ to $\tilde{f}\left(\mathbb{R}^{n}\right), f$ and $\widetilde{f}$ are Lag- $\mathcal{K}$-equivalent, by Lemma 8 below. Thus, by Lemma $7, f$ and $\widetilde{f}$ are Lag- $\mathcal{A}$-equivalent: For a $\left(\sigma, \tau_{3}\right) \in \operatorname{Lag}-\mathcal{A}, \tilde{f}=\tau_{3} \circ f \circ \sigma^{-1}$. Then $\tau_{2} \circ \tau_{3}$ preserves $\Lambda$ and $\pi^{\prime} \circ \tau_{2} \circ \tau_{3}=\pi$. Therefore $\pi$ and $\pi^{\prime}$ are $\Lambda$-equivalent.

\section{Simple stable compositions.}

Here we remark that our result is applied to the classification problem for compositions of an isotropic mapping and a Lagrange fibration [13], when the isotropic mapping is the first open Whitney umbrella. For this, first recall the notion of $C^{\infty}$ normalization $[6],[7]$ in the special case we need: 
A map-germ $f:\left(\mathbb{R}^{n}, 0\right) \rightarrow\left(\mathbb{R}^{p}, 0\right)$ is called a $C^{\infty}$ normalization if $f$ is $C^{\infty}$ right-left equivalent to an analytic map-germ $f^{\prime}:\left(\mathbb{R}^{n}, 0\right) \rightarrow\left(\mathbb{R}^{p}, 0\right)$ which is a normalization of the image.

Recall that a map-germ $f:\left(\mathbb{R}^{n}, 0\right) \rightarrow(E, 0)$ is called an open Whitney umbrella of type $k$ if $f$ is symplectically equivalent to a polynomial map-germ $f_{n, k}:\left(\mathbb{R}^{n}, 0\right) \rightarrow\left(T^{*} \mathbb{R}^{n}, 0\right)$ explicitly given in [13], namely $f \circ \sigma=\tau \circ f_{n, k}$ for a diffeomorphism $\sigma:\left(\mathbb{R}^{n}, 0\right) \rightarrow\left(\mathbb{R}^{n}, 0\right)$ and a symplectic diffeomorphism $\tau:(E, 0) \rightarrow(E, 0)$.

Remark that the normal form $f_{n, k}$ of open Whitney umbrellas is an analytic normalization of the image, and therefore any open Whitney umbrella is a $C^{\infty}$-normalization. The following lemma is a special case of Theorem 1.11 of $[7]$ :

Lemma 8. Let $f:\left(\mathbb{R}^{n}, 0\right) \rightarrow(E, 0)$ be a $C^{\infty}$ normalization. Denote by $f\left(\mathbb{R}^{n}\right)$ the well-defined germ of the image of $f$. If a germ of diffeomorphism $h:(E, 0) \rightarrow(E, 0)$ preserves $f\left(\mathbb{R}^{n}\right)$, namely if $h\left(f\left(\mathbb{R}^{n}\right)\right)=f\left(\mathbb{R}^{n}\right)$, then there exists a germ of diffeomorphism $\sigma:\left(\mathbb{R}^{n}, 0\right) \rightarrow\left(\mathbb{R}^{n}, 0\right)$ such that $h \circ f=f \circ \sigma$.

Then we have:

Lemma 9. Let $f, f^{\prime}:\left(\mathbb{R}^{n}, 0\right) \rightarrow(E, 0)$ be open Whitney umbrellas of same type $k$, and $\pi, \pi^{\prime}:(E, 0) \rightarrow(Y, 0)$ Lagrange fibrations. Then the following conditions are equivalent:

(1) There exist a diffeomorphism $\sigma:\left(\mathbb{R}^{n}, 0\right) \rightarrow\left(\mathbb{R}^{n}, 0\right)$, a symplectic diffeomorphism $\tau:(E, 0) \rightarrow(E, 0)$ and a diffeomorphism $\bar{\tau}:(Y, 0) \rightarrow(Y, 0)$ with $f^{\prime} \circ \sigma=\tau \circ f$ and $\pi^{\prime} \circ \tau=\bar{\tau} \circ \pi$. (Lagrange equivalence of $(f, \pi)$ and $\left(f^{\prime}, \pi^{\prime}\right)$, in the sense of $\left.[\mathbf{1 5}].\right)$

(2) There exists a symplectic diffeomorphism $\tau:(E, 0) \rightarrow(E, 0)$ and a diffeomorphism $\bar{\tau}:(Y, 0) \rightarrow(Y, 0)$ satisfying $\tau\left(f\left(\mathbb{R}^{n}\right)\right)=f^{\prime}\left(\mathbb{R}^{n}\right)$ and $\pi^{\prime} \circ \tau=\bar{\tau} \circ \pi$.

Proof. The implication (1) $\Rightarrow(2)$ is straightforward. $(2) \Rightarrow(1)$ : First assume $\pi^{\prime}=\pi$. Since both $f$ and $f^{\prime}$ are symplectically equivalent to the parametrization $f_{n, k}$, there exist diffeomorphisms $\sigma^{\prime}, \sigma^{\prime \prime}:\left(\mathbb{R}^{n}, 0\right) \rightarrow\left(\mathbb{R}^{n}, 0\right)$ and symplectic diffeomorphisms $\tau^{\prime}, \tau^{\prime \prime}:(E, 0) \rightarrow(E, 0)$ such that $f \circ \sigma^{\prime}=$ $\tau^{\prime} \circ f_{n, k}$ and $f^{\prime} \circ \sigma^{\prime \prime}=\tau^{\prime \prime} \circ f_{n, k}$. We set $\Lambda_{k}=f_{n, k}\left(\mathbb{R}^{n}\right)$. Then we see that $\tau^{\prime \prime-1} \circ \tau \circ \tau^{\prime}\left(\Lambda_{k}\right)=\Lambda_{k}$. By Lemma 8, there exists a diffeomorphism $\sigma^{\prime \prime \prime}:\left(\mathbb{R}^{n}, 0\right) \rightarrow\left(\mathbb{R}^{n}, 0\right)$ such that $\tau^{\prime \prime-1} \circ \tau \circ \tau^{\prime} \circ f_{n, k}=f_{n, k} \circ \sigma^{\prime \prime \prime}$. Then we have $\tau \circ f=f^{\prime} \circ \sigma^{\prime \prime} \circ \sigma^{\prime \prime \prime} \circ \sigma^{\prime-1}$.

In general case, apply the above argument to $f$ and $T \circ f^{\prime}$, for a symplectic diffeomorphism $T:(E, 0) \rightarrow(E, 0)$ satisfying $\pi^{\prime}=\pi \circ T([\mathbf{3}])$. 


\section{References}

[1] V.I. Arnol'd, Normal forms for functions near degenerate critical points, the Weyl groups of $A_{k}, D_{k}, E_{k}$ and Lagrangian singularities, Funct. Anal. Appl., 6(4) (1972), 254-272, MR 50 \#8595, Zbl 0278.57011.

[2] __ Lagrangian manifolds with singularities, asymptotic rays, and the open swallowtail, Funct. Anal. Appl., 15(4) (1981), 235-246, MR 83c:58011, Zbl 0484.58008.

[3] V.I. Arnol'd, S.M. Gusein-Zade and A.N. Varchenko, Singularities of Differentiable Maps I, Birkhäuser, 1985, MR 86f:58018, Zbl 0554.58001.

[4] I.A. Bogaevski, Singularities of convex hulls of three-dimensional hypersurfaces, Proc. Steklov Inst. Math., 221 (1998), 71-90.

[5] Singularities of linear waves in $2 D$ and $3 D$ space, Proceedings of Arnold's Seminar, AMS, forthcoming.

[6] A. du Plessis, T. Gaffney and L.C. Wilson, Map-germs determined by their discriminants, Stratifications, singularities and differential equations, I (Marseille, 1990; Honolulu, HI, 1990), 1-40, Travaux en Cours, 54, Hermann, Paris, 1997, MR 98k:58026, Zbl 0891.58006.

[7] T. Gaffney and L. Wilson, Equivalence of generic mappings and $C^{\infty}$ normalization, Compositio Math., 49(3) (1983), 291-308, MR 85g:58018, Zbl 0518.58009.

[8] A.B. Givental', Lagrangian imbeddings of surfaces and open Whitney umbrella, Funkt. Anal. Prilozhen, 20-3 (1986), 35-41; English transl., Funct. Anal. Appl., 20 (1986), 197-203, MR 88g:58018, Zbl 0621.58025.

[9] Singular Lagrangian manifolds and their Lagrangian mappings, Itogi Nauki Tekh., Ser. Sovrem. Prob. Mat. (Contemporary Problems of Mathematics), 33, VINITI, (1988), 55-112; English transl., J. Soviet Math., 52(4) (1990), 3246-3278, MR 91g:58077, Zbl 0731.58023.

[10] M. Golubitsky and V. Guillemin, Contact equivalence for Lagrangian manifolds, Adv. in Math., 15 (1975), 357-387, MR 51 \#1868, Zbl 0295.57018.

[11] G. Ishikawa, The local model of an isotropic map-germ arising from one dimensional symplectic reduction, Math. Proc. Camb. Phil. Soc., 111(1) (1992), 103-112, MR 93a:58022, Zbl 0761.58015.

[12] _ Transversalities for Lagrange singularities of isotropic mappings of corank one, in 'Singularities and Differential Equations', Banach Center Publications, 33 (1996), 93-104, MR 98d:58012, Zbl 0853.58018.

[13] Symplectic and Lagrange stabilities of open Whitney umbrellas, Invent. Math., 126(2) (1996), 215-234, MR 97h:58022, Zbl 0869.58002.

[14] Singularities of developable surfaces, London Mathematical Society Lecture Notes Series, 263 (1999), 403-418, MR 2000m:58067, Zbl 0963.58017.

[15] _ Determinacy, transversality and Lagrange stability, in 'Geometry and Topology of Caustics - Caustics' 98', Banach Center Publications, 50 (1999), 123-135, MR 2001a:58055.

[16] S. Janeczko, Generating families for images of Lagrangian submanifolds and open swallowtails, Math. Proc. Cambridge Philos. Soc., 100 (1986), 91-107, MR 87h:58022, Zbl 0635.58012.

[17] J.N. Mather, Stability of $C^{\infty}$ mappings I: The division theorem, Ann. of Math., 87 (1968), 89-104, MR 38 \#726, Zbl 0159.24902. 
II: Infinitesimally stability implies stability, Ann. of Math., 89 (1969), 254-291, MR 41 \#4582, Zbl 0177.26002.

III: Finitely determined map-germs, Publ. Math. I.H.E.S., 35 (1968), 127-156, MR 43 \#1215a, Zbl 0208.52001.

IV: Classification of stable germs by $\mathbb{R}$ algebras, Publ. Math. I.H.E.S., 37 (1970), 223-248, MR 43 \#1215b, Zbl 0202.55102.

V: Transversality, Adv. Math., 4 (1970), 301-336, MR 43 \#1215c, Zbl 0207.54303.

VI: The nice dimensions, Lecture Notes in Math., 192 (1972), 207-253, MR 45 \#2747, Zbl 0211.56105.

[18] A.Kh. Rakhimov, Singularities of Riemannian invariants, Funct. Anal. Appl., 27(1) (1992), 39-50, Zbl 0794.35025.

[19] O.P. Shcherbak, Projectively dual space curves and Legendre singularities, Trudy Tbiliss. Univ., 232-233 (1982), 280-336; English transl., Sel. Math. Sov., 5(4) (1986), 391-421, Zbl 0621.58029.

[20] _ Wavefront and reflection groups, Russian Math. Surveys, 43(3) (1988), 149194, Zbl 0675.58007.

[21] C.T.C. Wall, Finite determinacy of smooth map-germs, Bull. London Math. Soc., 13 (1981), 481-539, MR 83i:58020, Zbl 0459.58004.

[22] V.M. Zakalyukin, Generating ideals of Lagrangian varieties, Theory of Singularities and its Applications, ed. by V.I. Arnol'd, Advances in Soviet Mathematics, 1, Amer. Math. Soc., (1990), 201-210, MR 92g:58038, Zbl 0731.58025.

[23] Simple coisotropic caustics, preprint, Universite de Bourgogne, 189 (1999).

[24] V.M. Zakalyukin and O.M. Myasnichenko, Lagrange singularities under symplectic reduction, Funct. Anal. Appl., 32(1) (1998), 1-9, MR 99g:58056, Zbl 0919.57021.

[25] V.M. Zakalyukin and R.M. Roberts, On stable singular Lagrangian varieties, Funct. Anal. Appl., 26(3) (1992), 174-178, Zbl 0799.58033.

Received May 16, 2000 and revised February 1, 2001. The first author was partially supported by RFBR 99-01-01109 \& NWO-RFBR 047-008-005. The second author was partially supported by Grants-in-Aid for Scientific Research, The Ministry of Education, Science, Sports and Culture, Japan, No. 10440013.

\section{INDEPENDENT UNIVERSITY OF MOSCOW}

Bolshoi Vlas'evskit Per. 11, Moscow 121002

Russia

E-mail address: bogaevsk@mccme.ru

Department of Mathematics

HOKKAIDO UNIVERSITY

SAPPORO 060-0810, JAPAN

E-mail address: ishikawa@math.sci.hokudai.ac.jp 